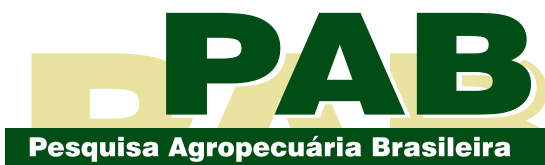

ISSN 1678-3921

Journal homepage: www.embrapa.br/pab

For manuscript submission and journal contents, access: www.scielo.br/pab

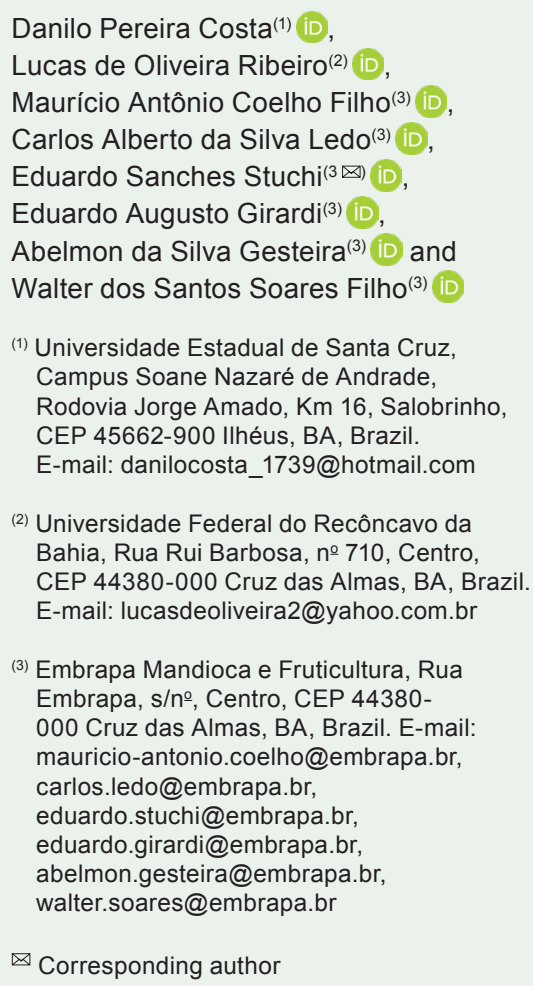

(2) Universidade Federal do Recôncavo da Bahia, Rua Rui Barbosa, № 710, Centro, CEP 44380-000 Cruz das Almas, BA, Brazil. E-mail: lucasdeoliveira2@yahoo.com.br

(3) Embrapa Mandioca e Fruticultura, Rua Embrapa, s/no, Centro, CEP 44380000 Cruz das Almas, BA, Brazil. E-mail: mauricio-antonio.coelho@embrapa.br, carlos.ledo@embrapa.br, eduardo.stuchi@embrapa.br, eduardo.girardi@embrapa.br. abelmon.gesteira@embrapa.br walter.soares@embrapa.br

$\bowtie$ Corresponding author

Received

November 14, 2019

Accepted

August 19, 2020

How to cite

COSTA, D.P.; RIBEIRO, L. de O.; COELHO FILHO, M.A.; LEDO, C.A. da S.; STUCHI, E.S.; GIRARDI, E.A.; GESTEIRA, A. da S.; SOARES FILHO, W. dos S. Nonparametric indices for the selection of hybrid citrus as rootstocks grafted with 'Valência' sweet orange. Pesquisa Agropecuária Brasileira, v.55, e01592, 2020. DOI: https://doi.org/10.1590/S1678-3921. pab2020.v55.01592.
Pomology/ Original Article

\section{Nonparametric indices for the selection of hybrid citrus as rootstocks grafted with 'Valência' sweet orange}

\begin{abstract}
The objective of this work was to evaluate five nonparametric selection indices for the selection of hybrid citrus rootstocks grafted with 'Valência' sweet orange, using horticultural traits relevant for the juice processing industry. Forty-six rootstocks were evaluated in a randomized complete block design, with three replicates and five trees in the plot, in the period from 2009-2015, in a rainfed cultivation. The means of the variables plant height, accumulated fruit yield, fruit yield efficiency, total soluble solids concentration, juice yield, and drought-tolerance were used to calculate the following indices: multiplicative index $\left(\mathrm{I}_{\mathrm{Ei}}\right)$, sum of classification $\left(\mathrm{I}_{\mathrm{MMi}}\right)$, genotype-ideotype distance $\left(\mathrm{D}_{\mathrm{il}}\right)$, and ranking indices $\left(\mathrm{I}_{\mathrm{RK}}\right.$, based on simple means; and $\mathrm{I}_{\mathrm{RKii}}$, based on linear normalization). The indices were efficient to classify the hybrids in relation to general performance. Spearman's correlation showed a high similarity between most nonparametric indices, notably between $I_{R K i}$ and $I_{R K i i}$. The ranking indices, mainly $I_{R K i i}$, provide a more coherent classification of the hybrids, which allows of the selection of more productive and drought-tolerant rootstocks to produce high-quality fruit for processing.
\end{abstract}

Index terms: Citrus, Poncirus trifoliata, breeding, genetic variability, selection index.

\section{Índices não paramétricos para seleção de citros híbridos como porta-enxertos, enxertados com laranjeira 'Valência'}

Resumo - O objetivo deste trabalho foi avaliar cinco índices de seleção não paramétricos para a seleção de citros híbridos como porta-enxertos, enxertados com laranjeira 'Valência', com base em atributos horticulturais relevantes para a indústria de processamento de suco. Avaliaram-se 46 portaenxertos em delineamento de blocos ao acaso, com três repetições e cinco plantas na parcela, no período de 2009-2015, em cultivo de sequeiro. As médias das variáveis altura de planta, produção acumulada de frutos, eficiência produtiva de frutos, concentração de sólidos solúveis totais, rendimento de suco e tolerância à seca foram utilizadas para calcular os seguintes índices: multiplicativo $\left(\mathrm{I}_{\mathrm{Ei}}\right)$, soma de classificação $\left(\mathrm{I}_{\mathrm{MMi}}\right)$, distância genótipo-ideótipo $\left(D_{\mathrm{iI}}\right)$ e ranqueamento $\left(\mathrm{I}_{\mathrm{RKi}}\right.$, baseado em médias simples; e $\mathrm{I}_{\mathrm{RKii}}$, baseado em normalização linear). Os índices foram eficientes em classificar os híbridos em relação ao desempenho geral. A correlação de Spearman mostrou alta similaridade entre a maioria dos índices não paramétricos, notadamente entre $\mathrm{I}_{\mathrm{RKi}}$ e $\mathrm{I}_{\mathrm{RKii}}$. Os índices de ranqueamento, principalmente o $\mathrm{I}_{\mathrm{RKii}}$, fornecem uma classificação mais coerente dos híbridos, o que permite a seleção de portaenxertos mais produtivos e tolerantes à seca, para a produção de frutas de alta qualidade para processamento.

Termos de indexação: Citrus, Poncirus trifoliata, melhoramento, variabilidade genética, índice de seleção. 


\section{Introduction}

Despite its socioeconomic importance, Brazilian citrus production is vulnerable to several abiotic and biotic stresses because of the low variability of the available genetic material, mainly of rootstock cultivars (Bastos et al., 2014). The citrus rootstock influences more than 20 characteristics of the scion variety, from drought-tolerance to pest resistance, tree size, and fruit traits (Castle, 2010). This fact poses a challenge for the appropriate selection of superior genotypes in breeding programs that, in turn, have been introducing hundreds of new hybrid rootstocks to promote varietal diversification (Soares Filho, 2012).

Traditionally, citrus rootstock breeding has been based on the massal selection of hybrid progenies obtained from crossing a female parental, preferably monoembryonic, which usually has lowerheterozygosis levels, and a male parental of Poncirus trifoliata (L.) Raf. (Schinor et al., 2013). However, due to the high genetic segregation of citrus (Navarro et al., 2002), obtaining hybrids that combine good performance in multiple traits is seldom an easy task. In this sense, nonparametric indices are auxiliary tools that involve the simultaneous combination of several attributes of interest, to allow a more efficient selection of promising genotypes (Vilarinho et al., 2003).

The nonparametric indices do not require the estimation of genetic parameters and can be used for random samples, selected genotypes, or hybrids, that is, fixed samples (Vilarinho et al., 2003). Some indices that are frequently used to assist the breeding of annual and perennial crops (Ferreira et al., 2005; Lessa et al., 2010; Dovale et al., 2011; Almeida et al., 2014; Lessa et al., 2017) are the following: the multiplicative index (Elston, 1963), the classification sum index (Mulamba $\&$ Mock, 1978), and the genotype-ideotype distance (Schwarzbach, 1972).

Few papers have reported the application of nonparametric indices to select citrus genotypes. Selection is commonly based on the empiric experience of the breeder, as well as on classic univariate analyses of extensive datasets and, in some cases, on multivariate analyses. However, the interest in indices to assist citrus breeding is increasing, as for instance, by the following authors: Caputo et al. (2012), who used a performance index to select early-ripening sweet orange cultivars; Yacomelo et al. (2018), who selected 'Margaritera' orange genotypes through an index based on fruit quality traits; and Costa et al. (2016), who classified hybrid citrus rootstocks according to a ranking index. Selection indices should consider variables that are highly relevant for the market acceptance, such as juice content and quality, fruit yield, a high-canopy production efficiency, dwarfism, and drought-tolerance (Auler et al., 2008; Tazima et al., 2008; Castle, 2010; Khalid et al., 2012). Therefore, different selection indices should be investigated for key traits, in order to assess and select citrus genotypes more precisely.

The objective of this work was to evaluate five nonparametric selection indices for the selection of hybrid citrus rootstocks grafted with 'Valência' sweet orange, using relevant horticultural traits for the juice processing industry.

\section{Materials and Methods}

The datasets come from an experiment that was planted in 2007, in the municipality of Colômbia, in the state of São Paulo, Brazil (20¹9'22"S, 48 41'10"W, at $492 \mathrm{~m}$ altitude). The scion cultivar was 'Valência' IAC sweet orange [Citrus sinensis (L.) Osbeck], which was grafted onto 46 citrus rootstocks, most of them were hybrids introduced or obtained by the Citrus Breeding Program of Embrapa Mandioca e Fruticultura, in the municipality of Cruz das Almas, in the state of Bahia, Brazil (Tables 1 to 5). 'Cravo Santa Cruz' Rangpur lime (Citrus limonia Osbeck) was the commercial standard rootstock. The experimental design was carried out in randomized complete blocks, with 46 treatments, three replicates, and five trees per plot.

The local climate type is Aw, according to the Köppen-Geiger's classification (hot rainy summer, and dry winter typical of savannah), with $1,322 \mathrm{~mm}$ mean annual rainfall, and $26.3^{\circ} \mathrm{C}$ mean annual air temperature (Cepagri, 2018). Tree spacing was $6.0 \times 2.5 \mathrm{~m}$, in a rainfed orchard on a Latossolo Vermelho escuro (Oxisol), medium texture, with moderate A layer. Crop management followed the standard recommendations for orange trees in São Paulo (Mattos Jr. et al., 2014).

In the period 2009-2017, trees were assessed annually for the following variables, which are the most important ones to the juice processing industry: accumulated fruit production (AP), determined by weighing fruit from all trees on a digital scale (kg per tree, in 2009-2015); mean canopy production efficiency (EF), calculated by the mean ratio between 
the annual production per tree and the annual canopy volume per tree in 2009-2015 $\left(\mathrm{kg} \mathrm{m}^{-3}\right)$, and volume calculated as described by Cantuarias-Avilés et al. (2011); mean soluble solids concentration in the juice (SS) in 2009-2015, measured ( ${ }^{\circ}$ Brix) with a refractometer Palette PR-101 (Atago, Tokyo, Japan); mean juice yield (JC) in 2009-2015, calculated by the ratio between juice weight and fruit weight (\%), after extraction in a semi-commercial apparatus Otto 1800 juice extractor (OIC, Limeira, SP, Brazil); mean tree height $(\mathrm{TH})$, measured with a ruler $(\mathrm{m})$ from the rootstock collar end to the canopy tip; and droughttolerance (DT), visually assessed from 2010 to 2017, except for 2015, using scores from 1 to 3 according to the intensity of leaf wilting during winter months (Stuchi et al., 2000; Schinor et al., 2013). Data were subjected to the analysis of variance, to obtain the coefficient of variation and significance ( $p<0.01$ and $\mathrm{p}<0.05$ ) of the variables used in the selection indices.

The multiplicative index $\left[\mathrm{I}_{\mathrm{Ei}}\right]$ (Elston, 1963) was calculated as

$I_{E i}=\log \prod_{j=1}^{m}\left(X_{i j}-k_{j}\right)=\log \left[\left(X_{i 1}-k_{1}\right)\left(X_{i 2}-k_{2}\right) \ldots\left(X_{i n}-k_{n}\right)\right]$,

in which: $I_{E \mathrm{i}}$ is the multiplicative index; $\mathrm{x}_{\mathrm{ij}}$ is the mean of the trait $\mathrm{j}$, measured in genotype $\mathrm{I}$; and $\mathrm{k}_{\mathrm{j}}$ is the lowest value to select

$\mathrm{K}_{\mathrm{j}}=\left(\frac{\mathrm{n}\left(\text { mín. }_{\mathrm{ij}}-\text { máx. }_{\mathrm{ij}}\right.}{\mathrm{n}-1}\right)$,

in which: $\mathrm{n}$ is the number of genotypes; and min. $\mathrm{x}_{\mathrm{ij}}$ and max. $\mathrm{x}_{\mathrm{ij}}$ are, respectively, the lowest and the highest mean of trait $\mathrm{j}$.

The classification sum index $\left[\mathrm{I}_{\mathrm{MMi}}\right]$ (Mulamba \& Mock, 1978) was calculated by

$\mathrm{I}_{\mathrm{MMi}}=\sum_{\mathrm{j}=1}^{\mathrm{m}} \mathrm{n}_{\mathrm{ij}}$

in which: $\mathrm{I}_{\mathrm{MMi}}$ is the classification sum indices; and $\mathrm{n}_{\mathrm{ij}}$ is the number of classifications of genotype $i$ in relation to trait $\mathrm{j}$.

The ranking index $\left(\mathrm{I}_{\mathrm{RK}}\right)$ was modified from Costa et al. (2016), and calculated by

$\mathrm{I}_{\mathrm{RK}}=(\mathrm{AP} \times 0.2)+(\mathrm{EF} \times 0.2)+(\mathrm{SS} \times 0.15)+(\mathrm{JC} \times 0.15)+$ $(\mathrm{DT} \times 0.2)+((1 / \mathrm{TH}) \times 0.1)$, in which: AP is the accumulated production; EF is the canopy production efficiency; SS represents the concentration of soluble solids; JC is the juice yield; DT is the drought-tolerance; and TH is the tree height.

The weights of each variable were determined according to their relevance from researcher experience. $I_{R K}$ was calculated in two manners: $I_{R K i}$, using the weight sum equal to 1 (simple means of data); and $\mathrm{I}_{\mathrm{RKii}}$ - whose data were subjected to linear normalization for the interval $[0,1])$ - was determined by $\mathrm{F}\left(\mathrm{x}_{\mathrm{i}}\right)=\left(\mathrm{x}_{\mathrm{i}}-\mathrm{x}_{\min }\right) /\left(\mathrm{x}_{\max }-\mathrm{x}_{\min }\right)$, where $\mathrm{x}_{\mathrm{i}}$ is the numerical value of the variable for each rootstock, and $x_{\min }$ and $\mathrm{x}_{\max }$ are the minimum and maximum values of each variable.

The genotype-ideotype distance index $\left[\mathrm{D}_{\mathrm{iI}}\right]$ was determined (Schwarzbach, 1972) by the Euclidean distance according to the following equation:

$\mathrm{D}_{\mathrm{iI}}=\sqrt{\sum_{\mathrm{j}=1}^{\mathrm{m}} \mathrm{d}_{\mathrm{ij}}^{2}}$

in which: $D_{i 1}$ is the Euclidean distance between genotype $\mathrm{i}$ and ideotype $\mathrm{I}$; and $\mathrm{d}_{\mathrm{ij}}$ is the standard deviation between the mean of trait $j$, measured in genotype $\mathrm{i}\left(\mathrm{x}_{\mathrm{ij}}\right)$, and the value given to the ideotype for this trait $\left(\mathrm{x}_{\mathrm{Ij}}\right)$, that is, $\mathrm{d}_{\mathrm{ij}}=\left(\mathrm{x}_{\mathrm{ij}}-\mathrm{x}_{\mathrm{Ij}}\right) / \sigma_{\mathrm{j}}$. The standardization prevents traits measured in greater units from having greater influence than other traits on the value of the indices, and, consequently, on the genotype classification (Lessa et al., 2017).

The values given to the ideotype were based on information provided by juice processors, according to our experience, as follows: $\mathrm{SS}>11^{\circ} \mathrm{Brix}, \mathrm{JC}>50 \%$, $\mathrm{AP}>250 \mathrm{~kg}$ per tree, EF $>4 \mathrm{~kg} \mathrm{~m}^{-3}$, DT $\geq 2$, and $\mathrm{TH}<3 \mathrm{~m}$. The weight given to each variable in the formula followed the empirical relative importance of the variable for the selection, as $\mathrm{AP}=\mathrm{EF}>\mathrm{SS}=\mathrm{JC}>$ DT $>$ TH.

After the calculation of the indices, the genotypes were classified according to the recommendations of Garcia \& Souza Júnior (1999). Spearman correlation coefficients among the evaluated indices were calculated to observe the degree of agreement; and the significance of the estimates was tested at $1 \%$ and $5 \%$ probabilities (Costa Neto, 2002). 


\section{Results and Discussion}

All assessed variables showed significant differences, which allowed of the ranking of the hybrid citrus rootstocks, therefore confirming the variability within the evaluated genotypes. The coefficient of variation $(\mathrm{CV})$ of the variables used to calculate the indices were AP (16.13\%), EF (20.37\%), SS (3.37\%), JC (4.13\%), DT $(10.37 \%)$, and TH $(6.33 \%)$ (Table 1). Not only this wide genetic variability reflects the diverse parental background of the evaluated genotypes, but it is also commonly reported within populations of hybrid citrus rootstocks (Raga et al., 2012; Schinor et al., 2013).

The application of the $\mathrm{I}_{\mathrm{Ei}}$ to the dataset of the evaluated variables indicated that $52.17 \%$ of the hybrid citrus rootstocks were superior to the standard 'Santa Cruz' Rangpur lime (25 ${ }^{\text {th }}$ position), which was the Rangpur lime with highest position. The genotypes in the five first positions in the ranking were TSKC $\times$ $(\mathrm{LCR} \times \mathrm{TR})-059\left(1^{\text {st }}\right), \mathrm{TSKC} \times \mathrm{CTQT} 1434-010\left(2^{\text {nd }}\right)$, TSKC $\times($ LCR $\times$ TR $)-017\left(3^{\text {rd }}\right)$, TSK $\times$ TR 'Benecke' $-\mathrm{CO}\left(4^{\text {th }}\right)$ and 'San Diego' citrandarin $\left(5^{\text {th }}\right)$. Therefore, the $\mathrm{I}_{\mathrm{Ei}}$ led to the selection of rootstocks that combined lower-tree height $(3.14 \mathrm{~m})$, intermediate accumulated production (253.8 $\mathrm{kg}$ per tree), high-production efficiency $\left(3.68 \mathrm{~kg} \mathrm{~m}^{-3}\right)$, good drought-tolerance (1.98), high-SS (11.88 ${ }^{\circ}$ Brix), and juice yield close to that of the standard genotype (48.23\%) (Table 1).For the $\mathrm{I}_{\mathrm{MMi}}$, the best ranked rootstocks were TSKC $\times($ LCR $\times$ TR $)-$ $059\left(1^{\text {st }}\right)$, TSKC $\times$ CTQT $1434-010\left(2^{\text {nd }}\right)$, TSKC $\times($ LCR $x$ TR $)-017\left(3^{\text {rd }}\right)$, TSK $\times$ TR 'Benecke' - CO $\left(4^{\text {th }}\right)$ and LCR $\times$ TR $-001\left(5^{\text {th }}\right)$, in comparison to 'Cravo Santa Cruz' Rangpur lime (11 $\left.{ }^{\text {st }}\right)$ (Table 2). Therefore, the $\mathrm{I}_{\mathrm{MMi}}$ led to the selection of rootstocks combining lowertree height $(3.07 \mathrm{~m})$, intermediate accumulated yield (243.15 kg per tree), high-yield efficiency $\left(3.81 \mathrm{~kg} \mathrm{~m}^{-3}\right)$, good drought-tolerance (2.02), high-SS (11.79 ${ }^{\circ}$ Brix), and juice yield close to that of the standard genotype $(47.82 \%)$. This ranking prioritized the concentration of soluble solids and the production efficiency in a similar way to that obtained with $\mathrm{I}_{\mathrm{E}}$; however, some selected hybrids showed low yield due to their smaller tree size. By contrast, large-size inducing rootstocks led to low-production efficiency (Cantuarias-Avilés et al., 2011). Lessa et al. (2010) studied diploid banana hybrids, and they pointed out that the multiplicative index $\left(\mathrm{I}_{\mathrm{Ei}}\right)$ and the classification sum index $\left(\mathrm{I}_{\mathrm{MMi}}\right)$ also provided an adequate selection with high correlation, which allowed of a better adequacy of the results that helped with decision making.

The index $\mathrm{I}_{\mathrm{RKi}}$ classified the following rootstocks as the best ones: 'Indio' citrandarin $\left(1^{\text {st }}\right)$, 'Sunki' $\times$ 'English Palmira' - CO (2 $\left.{ }^{\text {nd }}\right)$, 'San Diego' citrandarin $\left(3^{\text {rd }}\right)$, CNPMF -004 Rangpur lime $\left(4^{\text {th }}\right)$, and TSKC $\times$ CTSW $-028\left(5^{\text {th }}\right)$, which ranked ahead of the standard 'Cravo Santa Cruz' Rangpur lime ( $\left.8^{\text {th }}\right)$ (Table 3). On average, these indices clearly selected rootstocks with higher-accumulated production (308.7 $\mathrm{kg}$ per tree), tall trees $(3.7 \mathrm{~m})$ with good drought-tolerance (2.0), and juice quality (11.75 ${ }^{\circ}$ Brix), but with lower efficiency $\left(3.18 \mathrm{~kg} \mathrm{~m}^{-3}\right)$. Or else, when normalized means were used, $\mathrm{I}_{\text {RKii }}$ selected the following genotypes: 'Sunki' $\times$ 'English Palmira' - CO (1 $\left.{ }^{\text {st }}\right)$, TSKC $\times($ LCR x TR $)-059$ $\left(2^{\text {nd }}\right)$, 'Indio' citrandarin $\left(3^{\text {rd }}\right)$, 'San Diego' citrandarin $\left(4^{\text {th }}\right)$, and CNPMF $-004\left(5^{\circ}\right)$, in comparison to 'Cravo Santa Cruz' Rangpur lime $\left(7^{\text {th }}\right)$ (Table 4). Therefore, in relation to $I_{R K i}$, the index $I_{R K i i}$ led to the selection of rootstocks that combined high-accumulated production (301.84 $\mathrm{kg}$ per tree), intermediate to large tree height $(3.57 \mathrm{~m})$, better production efficiency (3.4 $\left.\mathrm{kg} \mathrm{m}^{-3}\right)$, good drought-tolerance (2.04), high-SS $\left(11.82{ }^{\circ} \mathrm{Brix}\right)$, and juice yield near that of the standard genotype $(48.03 \%)$. For both ranking indices $\left(\mathrm{I}_{\mathrm{RK}}\right)$, rootstocks with low yield and low-drought-tolerance, such as 'Sunki' $\times$ 'Alemow' $-\mathrm{CO}$, were in the last positions. Caputo et al. (2012) also reported that a selection index using normalized data of phenotypic variables was useful to assess sweet orange varieties that were more promising for both fresh marketing and juice processing.

The rootstocks TSKC $\times$ CTARG $-001\left(1^{\text {st }}\right)$, 'Riverside' citrandarin ( $\left.2^{\text {nd }}\right)$, CNPMF - 004 Rangpur lime $\left(3^{\text {rd }}\right)$, 'Sunki' $\times$ 'English Palmira' - CO $\left(4^{\text {th }}\right)$, and 'Indio' citrandarin $\left(5^{\text {th }}\right)$ were superior to 'Cravo Santa Cruz' Rangpur lime $\left(9^{\text {th }}\right)$ by the $\mathrm{D}_{\text {iI }}$ (Table 5). On average, the selected rootstocks showed intermediate to high-accumulated yield (278.3 kg per tree), but with tall trees $(3.83 \mathrm{~m})$ and low-productive efficiency $(2.68$ $\left.\mathrm{kg} \mathrm{m}^{-3}\right)$. As to the ideotype used, 33, 13, 87, and 22\% of the evaluated hybrid citrus showed higher means for AP, EF, SS, and DT, respectively, but all them had lower JC.

In each index ranking, the averages of the first five rootstocks were highlighted because they accounted for around $10 \%$ of the selection pressure on the 46 evaluated genotypes. 'Indio' and 'San Diego' citrandarin, 'Sunki'

Pesq. agropec. bras., Brasília, v.55, e01592, 2020

DOI: 10.1590/S1678-3921.pab2020.v55.01592 
Table 1. Original and centered $\left(\mathrm{x}_{\mathrm{ij}}-\mathrm{k}_{\mathrm{j}}\right)$ means of the accumulated production per tree (AP), canopy production efficiency (EF), concentration of soluble solids (SS), juice yield (JC), drought-tolerance (DT) (visual scoring of leaf wilting), and tree height $(\mathrm{TH})$, to calculate the multiplicative index $\left(\mathrm{I}_{\mathrm{E}}\right)$ for the classification of hybrid citrus rootstocks grafted with 'Valência' sweet orange, in the north of São Paulo state, Brazil, 2009-2017.

\begin{tabular}{|c|c|c|c|c|c|c|c|c|c|c|c|c|c|c|}
\hline Rootstock & $\begin{array}{c}\text { AP } \\
\text { (kg per tree) }\end{array}$ & $x_{i j}-k_{j}$ & $\begin{array}{c}\mathrm{EF} \\
\left(\mathrm{kg} \mathrm{m}^{-3}\right)\end{array}$ & $\mathrm{x}_{\mathrm{ij}}-\mathrm{k}_{\mathrm{j}}$ & $\begin{array}{c}\text { SS } \\
\left({ }^{\circ} \text { Brix }\right)\end{array}$ & $\mathrm{x}_{\mathrm{ij}}-\mathrm{k}_{\mathrm{j}}$ & $\begin{array}{c}\mathrm{JC} \\
(\%)\end{array}$ & $\mathrm{x}_{\mathrm{ij}}-\mathrm{k}_{\mathrm{j}}$ & DT & $\mathrm{x}_{\mathrm{ij}}-\mathrm{k}_{\mathrm{j}}$ & $\begin{array}{l}\mathrm{TH} \\
(\mathrm{m})\end{array}$ & $\mathrm{x}_{\mathrm{ij}}-\mathrm{k}_{\mathrm{j}}$ & $\mathrm{I}_{\mathrm{Ei}}$ & Rank \\
\hline$\overline{T S K C} \times($ LCR $\times$ TR $)-059$ & 262.34 & 1.60 & 4.20 & 1.69 & 12.02 & 1.50 & 47.68 & 1.56 & 2.22 & 1.70 & 3.16 & 1.49 & 15.970 & 1 \\
\hline TSKC $\times$ CTQT $1434-010$ & 215.03 & 1.47 & 3.75 & 1.63 & 11.94 & 1.48 & 48.72 & 1.66 & 1.79 & 1.56 & 2.93 & 1.61 & 14.650 & 2 \\
\hline $\mathrm{TSKC} \times(\mathrm{LCR} \times \mathrm{TR})-017$ & 275.88 & 1.63 & 4.08 & 1.67 & 11.58 & 1.35 & 47.43 & 1.54 & 1.96 & 1.62 & 3.13 & 1.50 & 13.812 & 3 \\
\hline TSK $\times$ TR Benecke $-\mathrm{CO}$ & 210.47 & 1.45 & 2.88 & 1.46 & 12.50 & 1.61 & 48.10 & 1.61 & 1.92 & 1.61 & 3.12 & 1.51 & 13.350 & 4 \\
\hline San Diego citrandarin & 305.22 & 1.68 & 3.51 & 1.60 & 11.38 & 1.27 & 49.23 & 1.70 & 1.99 & 1.63 & 3.35 & 1.37 & 12.846 & 5 \\
\hline TSKC $\times$ CTSW -041 & 272.91 & 1.62 & 3.76 & 1.63 & 11.29 & 1.22 & 47.96 & 1.59 & 2.08 & 1.66 & 3.24 & 1.44 & 12.258 & 6 \\
\hline $\mathrm{TSKC} \times(\mathrm{LCR} \times \mathrm{TR})-001$ & 241.65 & 1.55 & 3.76 & 1.63 & 11.75 & 1.42 & 46.48 & 1.41 & 1.51 & 1.40 & 2.97 & 1.59 & 11.241 & 7 \\
\hline LRF $\times($ LCR $\times$ TR $)-005$ & 265.43 & 1.60 & 2.57 & 1.37 & 11.94 & 1.48 & 47.22 & 1.51 & 1.87 & 1.59 & 3.31 & 1.40 & 10.900 & 8 \\
\hline TSKC $\times$ CTSW -033 & 208.00 & 1.44 & 3.62 & 1.61 & 11.59 & 1.36 & 48.27 & 1.62 & 1.46 & 1.36 & 3.07 & 1.54 & 10.745 & 9 \\
\hline $\mathrm{LCR} \times \mathrm{TR}-001$ & 252.05 & 1.57 & 4.14 & 1.68 & 10.94 & 0.96 & 47.15 & 1.50 & 2.23 & 1.70 & 3.00 & 1.57 & 10.266 & 10 \\
\hline Sunki $\times$ English Palmira $-\mathrm{CO}$ & 317.99 & 1.70 & 2.90 & 1.47 & 12.88 & 1.68 & 47.85 & 1.58 & 1.93 & 1.61 & 3.76 & 0.93 & 9.904 & 11 \\
\hline $\mathrm{HTR}-053$ & 287.26 & 1.65 & 3.87 & 1.65 & 11.27 & 1.21 & 45.94 & 1.32 & 2.05 & 1.65 & 3.34 & 1.37 & 9.869 & 12 \\
\hline TSKC x CTSW - 028 & 296.79 & 1.66 & 3.14 & 1.53 & 11.68 & 1.39 & 47.30 & 1.52 & 2.02 & 1.64 & 3.70 & 1.02 & 9.020 & 13 \\
\hline TSKC x CTQT $1439-026$ & 188.45 & 1.35 & 3.54 & 1.60 & 12.23 & 1.55 & 48.14 & 1.61 & 1.19 & 1.00 & 2.87 & 1.64 & 8.806 & 14 \\
\hline CLEO $x$ TR Rubidoux - CO & 151.20 & 1.04 & 2.45 & 1.32 & 12.99 & 1.70 & 47.93 & 1.59 & 1.49 & 1.39 & 2.72 & 1.70 & 8.755 & 15 \\
\hline HTR -051 & 244.33 & 1.56 & 3.48 & 1.59 & 11.58 & 1.36 & 45.21 & 1.16 & 1.78 & 1.55 & 3.28 & 1.42 & 8.559 & 16 \\
\hline CLEO x CTCZ - 226 & 230.46 & 1.52 & 3.06 & 1.51 & 11.77 & 1.43 & 45.40 & 1.21 & 1.52 & 1.41 & 3.22 & 1.45 & 8.057 & 17 \\
\hline Indio citrandarin & 320.96 & 1.70 & 3.17 & 1.53 & 11.58 & 1.36 & 48.01 & 1.60 & 1.91 & 1.61 & 3.78 & 0.89 & 8.051 & 18 \\
\hline Sunki Tropical mandarin & 291.21 & 1.65 & 2.72 & 1.42 & 11.52 & 1.33 & 48.09 & 1.61 & 1.97 & 1.62 & 3.73 & 0.97 & 7.883 & 19 \\
\hline $\mathrm{TSKC} \times(\mathrm{LCR} \times \mathrm{TR})-073$ & 253.95 & 1.58 & 3.32 & 1.56 & 11.67 & 1.39 & 45.45 & 1.22 & 1.67 & 1.50 & 3.49 & 1.25 & 7.837 & 20 \\
\hline TSKC $\times$ CTSW -064 & 205.34 & 1.43 & 3.92 & 1.65 & 11.45 & 1.30 & 45.92 & 1.32 & 1.35 & 1.25 & 3.08 & 1.53 & 7.828 & 21 \\
\hline TSKFL $\times$ CTC $25-010$ & 197.38 & 1.40 & 3.76 & 1.63 & 11.42 & 1.29 & 45.11 & 1.13 & 1.46 & 1.36 & 2.87 & 1.64 & 7.355 & 22 \\
\hline TSKC $\times$ CTQT $1439-004$ & 208.50 & 1.44 & 2.09 & 1.10 & 11.55 & 1.34 & 48.51 & 1.64 & 1.70 & 1.52 & 3.34 & 1.37 & 7.333 & 23 \\
\hline TSKC $\times$ LHA -006 & 211.22 & 1.45 & 2.22 & 1.20 & 11.69 & 1.40 & 48.15 & 1.61 & 1.50 & 1.40 & 3.41 & 1.32 & 7.250 & 24 \\
\hline Cravo Santa Cruz Rangpur lime & 276.19 & 1.63 & 3.41 & 1.58 & 10.85 & 0.86 & 49.07 & 1.69 & 2.03 & 1.65 & 3.59 & 1.15 & 7.060 & 25 \\
\hline TSKFL $\times$ CTTR -012 & 193.10 & 1.38 & 3.52 & 1.60 & 10.98 & 1.00 & 46.40 & 1.40 & 1.65 & 1.49 & 3.11 & 1.52 & 6.948 & 26 \\
\hline CNPI & 239.38 & 1.54 & 3.33 & 1.56 & 10.81 & 0.80 & 47.10 & 1.50 & 2.08 & 1.66 & 3.26 & 1.43 & 6.880 & 27 \\
\hline CNPMF - 004 Rangpur lime & 302.69 & 1.67 & 3.19 & 1.54 & 11.23 & 1.19 & 47.36 & 1.53 & 2.15 & 1.68 & 3.81 & 0.83 & 6.526 & 28 \\
\hline TSK $\times$ CTTR -002 & 218.36 & 1.48 & 2.76 & 1.43 & 11.15 & 1.13 & 44.99 & 1.09 & 2.16 & 1.68 & 3.31 & 1.40 & 6.150 & 29 \\
\hline $\mathrm{LVK} \times \mathrm{LCR}-010$ & 242.26 & 1.55 & 3.33 & 1.56 & 10.99 & 1.01 & 44.98 & 1.09 & 1.77 & 1.55 & 3.20 & 1.47 & 6.057 & 30 \\
\hline TSKFL $\times$ CTTR -022 & 161.02 & 1.16 & 2.88 & 1.47 & 11.56 & 1.35 & 47.55 & 1.55 & 1.19 & 1.00 & 2.87 & 1.64 & 5.821 & 31 \\
\hline $\mathrm{TSKC} \times(\mathrm{LCR} \times \mathrm{TR})-018$ & 213.01 & 1.46 & 3.31 & 1.56 & 11.04 & 1.05 & 45.25 & 1.17 & 1.48 & 1.38 & 3.13 & 1.50 & 5.799 & 32 \\
\hline & 237.46 & 1.54 & 4.28 & 1.69 & 11.37 & 1.26 & 44.12 & 0.62 & 2.03 & 1. & 2.93 & 1.61 & 5.3 & 33 \\
\hline Riverside ci & 251.01 & 1.57 & 2.47 & 1.33 & 11.65 & 1.38 & 46.50 & 1.42 & 1.96 & 1.62 & 3.82 & 0.81 & 5.374 & 34 \\
\hline LVK $\times$ LVA -009 & 215.68 & 1.47 & 2.72 & 1.42 & 12.39 & 1.59 & 44.21 & 0.70 & 1.77 & 1.55 & 3.27 & 1.42 & 5.120 & 35 \\
\hline TSKC $\times$ CTARG -036 & 171.80 & 1.25 & 2.26 & 1.23 & 11.47 & 1.31 & 45.58 & 1.25 & 1.29 & 1.18 & 3.21 & 1.46 & 4.322 & 36 \\
\hline LCREEL $\times$ CTSW - 001 & 149.48 & 1.02 & 2.51 & 1.34 & 12.07 & 1.51 & 44.82 & 1.03 & 1.36 & 1.26 & 3.22 & 1.46 & 3.931 & 37 \\
\hline TSKC $\times$ LHA -011 & 172.43 & 1.25 & 1.95 & 0.97 & 11.98 & 1.49 & 45.00 & 1.09 & 1.58 & 1.45 & 3.43 & 1.30 & 3.725 & 38 \\
\hline TSKC & 145.00 & 0.95 & 2.00 & 1.02 & 12.06 & 1.51 & 47.03 & 1.49 & 1.07 & 0 . & 3.32 & 1.3 & 1.576 & 39 \\
\hline Sunki Maravilha mandarin & 174.12 & 1.26 & 1.72 & 0.44 & 11.79 & 1.43 & 45.80 & 1.30 & 1.08 & 0.55 & 3.34 & 1.37 & 0.783 & 40 \\
\hline $\mathrm{HTR}-116$ & 246.13 & 1.56 & 4.07 & 1.67 & 11.32 & 1.24 & 43.80 & 0.06 & 1.46 & 1.36 & 3.06 & 1.54 & 0.418 & 41 \\
\hline HTR - 069 & 215.20 & 1.47 & 4.33 & 1.70 & 10.58 & 0.04 & 46.05 & 1.34 & 1.85 & 1.58 & 3.07 & 1.54 & 0.301 & 42 \\
\hline TSKC $\times$ CTSW -019 & 235.69 & 1.53 & 2.02 & 1.04 & 11.51 & 1.33 & 43.82 & 0.12 & 1.27 & 1.15 & 3.73 & 0.98 & 0.282 & 43 \\
\hline TSKFL $\times$ CTTR -008 & 157.45 & 1.12 & 2.52 & 1.35 & 12.09 & 1.52 & 43.80 & 0.04 & 1.22 & 1.05 & 3.04 & 1.55 & 0.139 & 44 \\
\hline Sunki $\times$ Alemow $-\mathrm{CO}$ & 124.79 & 0.04 & 2.62 & 1.39 & 11.34 & 1.25 & 45.65 & 1.27 & 1.04 & 0.04 & 3.01 & 1.57 & 0.005 & 45 \\
\hline TSKC $\times$ CTARG -001 & 198.78 & 1.40 & 1.66 & 0.04 & 11.67 & 1.39 & 44.42 & 0.85 & 1.23 & 1.08 & 3.98 & 0.04 & 0.002 & 46 \\
\hline Minimum & 124.79 & & 1.66 & & 10.58 & & 43.80 & & 1.04 & & 2.72 & & & \\
\hline Maximum & 320.96 & & 4.33 & & 12.99 & & 49.23 & & 2.23 & & 3.98 & & & \\
\hline $\mathrm{Kj}$ & 116.81 & & 0.57 & & 9.29 & & 41.73 & & -0.01 & & 1.64 & & & \\
\hline $\mathrm{F}$ & $* *$ & & $* *$ & & $* *$ & & $* *$ & & $* *$ & & $* *$ & & & \\
\hline Coefficient of variation (\%) & 16.55 & & 20.37 & & 3.37 & & 4.13 & & 10.37 & & 6.32 & & & \\
\hline Mean & 227.28 & & 3.10 & & 11.61 & & 46.49 & & 1.68 & & 3.27 & & & \\
\hline
\end{tabular}

TSK, 'Sunki' mandarin [Citrus sunki (Hayata) hort. ex Tanaka]; TSKC, common 'Sunki' mandarin (C. sunki); TR, trifoliate orange [Poncirus trifoliata (L.) Raf.]; LCR, Rangpur lime (C. limonia Osbeck); CTSW, 'Swingle' citrumelo (C. paradisi Macfad. x P. trifoliata); HTR, trifoliate hybrid (P. trifoliata x sp.); LRF, 'Florida' rough lemon (C. jambhiri Lush.); CTQT, 'Thomasville' citrangequat [Fortunella margarita (Lour.) Swingle x 'Willits' citrange]; LHA, 'Hamlin' sweet orange [C. sinensis (L.) Osbeck]; CTCZ, 'Carrizo' citrange (C. sinensis x P. trifoliata); CTTR, 'Troyer' citrange (C. sinensis x P. trifoliata); LVK, 'Volkamer' lemon (C. volkameriana (Risso) V. Ten. \& Pasq.); LVA, 'Valência' sweet orange (C. sinensis); TSKFL, 'Florida Sunki' mandarin (C. sunki); CTC, citrange (C. sinensis x P. trifoliata); CLEO, 'Cleópatra' mandarin (C. reshni hort. ex Tanaka); CTARG, 'Argentina' citrange (C. sinensis x P. trifoliata); LCREEL, 'Santa Cruz' Rangpur lime (C. Limonia); CTRK, 'Rusk' citrange (C. sinensis x P. trifoliata); 'Alemow' (C. macrophylla Wester); English Palmira - CO: Sunki x English Palmira - CO is a selection of a USDA hybrid (C. sunki x P. trifoliata cv. English) introduced from the city of Palmira in Colombia; CNPMF, Embrapa Mandioca e Fruticultura. **Significant at $1 \%$ probability. The calculation of the multiplicative index was based on the methodology described by Elston (1963), where, $\mathrm{x}_{\mathrm{ij}}$ is the mean of the character $\mathrm{j}$, measured in the genotype $\mathrm{i}$, and $\mathrm{k}_{\mathrm{j}}$ is the lowest selectable value. 
Table 2. Original means of the accumulated production (AP), canopy production efficiency (EF), soluble solids concentration (SS), juice yield (JC), drought-tolerance (DT) (visual scoring of leaf wilting), and tree height (TH) for the determination of the classification sum index $\left(\mathrm{I}_{\mathrm{MMi}}\right)$, to rank the hybrid citrus rootstocks grafted with 'Valência' sweet orange, in the north of São Paulo state, Brazil, 2009-2017.

\begin{tabular}{|c|c|c|c|c|c|c|c|c|c|c|c|c|c|c|}
\hline Rootstock & $\begin{array}{c}\mathrm{AP} \\
\text { (kg per tree) }\end{array}$ & Rank & $\begin{array}{c}\mathrm{EF} \\
\left(\mathrm{kg} \mathrm{m}^{-3}\right)\end{array}$ & Rank & $\begin{array}{c}\text { SS } \\
\left({ }^{\circ} \text { Brix }\right) \\
\end{array}$ & Rank & $\begin{array}{l}\mathrm{JC} \\
(\%) \\
\end{array}$ & Rank & DT & Rank & $\begin{array}{l}\mathrm{TH} \\
(\mathrm{m}) \\
\end{array}$ & Rank & $\mathrm{I}_{\mathrm{MMi}}$ & Rank \\
\hline TSKC $\times($ LCR $\times$ TR $)-059$ & 262.34 & 12 & 4.20 & 3 & 12.02 & 9 & 47.68 & 14 & 2.22 & 2 & 3.16 & 19 & 59.000 & 1 \\
\hline TSKC $\times$ CTQT $1434-010$ & 215.03 & 27 & 3.75 & 9 & 11.94 & 12 & 48.72 & 3 & 1.79 & 20 & 2.93 & 6 & 77.000 & 2 \\
\hline TSKC $\times($ LCR $\times$ TR $)-017$ & 275.88 & 9 & 4.08 & 1 & 11.58 & 24 & 47.43 & 16 & 1.96 & 13 & 3.13 & 17 & 80.000 & 3 \\
\hline TSK $\times$ TR Benecke $-\mathrm{CO}$ & 210.47 & 30 & 2.88 & 15 & 12.50 & 3 & 48.10 & 8 & 1.92 & 16 & 3.12 & 16 & 88.000 & 4 \\
\hline $\mathrm{LCR} \times \mathrm{TR}-001$ & 252.05 & 14 & 4.14 & 4 & 10.94 & 43 & 47.15 & 20 & 2.23 & 1 & 3.00 & 8 & 90.000 & 5 \\
\hline TSKC $\times$ CTSW -041 & 272.91 & 10 & 3.76 & 7 & 11.29 & 36 & 47.96 & 11 & 2.08 & 5 & 3.24 & 24 & 93.000 & 6 \\
\hline San Diego citrandarin & 305.22 & 3 & 3.51 & 17 & 11.38 & 32 & 49.23 & 1 & 1.99 & 11 & 3.35 & 34 & 98.000 & 7 \\
\hline Sunki $\times$ English Palmira $-\mathrm{CO}$ & 317.99 & 2 & 2.90 & 25 & 12.88 & 2 & 47.85 & 13 & 1.93 & 15 & 3.76 & 42 & 99.000 & 8 \\
\hline TSKC $\times($ LCR $x$ TR $)-001$ & 241.65 & 19 & 3.76 & 10 & 11.75 & 15 & 46.48 & 24 & 1.51 & 29 & 2.97 & 7 & 104.000 & 9 \\
\hline $\mathrm{TSKC} \times \mathrm{CTSW}-028$ & 296.79 & 5 & 3.14 & 23 & 11.68 & 17 & 47.30 & 18 & 2.02 & 10 & 3.70 & 39 & 112.000 & 10 \\
\hline Cravo Santa Cruz Rangpur lime & 276.19 & 8 & 3.41 & 13 & 10.85 & 44 & 49.07 & 2 & 2.03 & 8 & 3.59 & 38 & 113.000 & 11 \\
\hline Indio citrandarin & 320.96 & 1 & 3.17 & 21 & 11.58 & 23 & 48.01 & 10 & 1.91 & 17 & 3.78 & 43 & 115.000 & 12 \\
\hline HTR -053 & 287.26 & 7 & 3.87 & 6 & 11.27 & 37 & 45.94 & 27 & 2.05 & 7 & 3.34 & 31 & 115.000 & 12 \\
\hline $\mathrm{LVK} \times \mathrm{LCR}-038$ & 237.46 & 21 & 4.28 & 5 & 11.37 & 33 & 44.12 & 43 & 2.03 & 9 & 2.93 & 5 & 116.000 & 14 \\
\hline $\mathrm{TSKC} \times \mathrm{CTSW}-033$ & 208.00 & 32 & 3.62 & 12 & 11.59 & 21 & 48.27 & 5 & 1.46 & 33 & 3.07 & 13 & 116.000 & 14 \\
\hline TSKC $\times$ CTQT $1439-026$ & 188.45 & 37 & 3.54 & 26 & 12.23 & 5 & 48.14 & 7 & 1.19 & 43 & 2.87 & 4 & 122.000 & 16 \\
\hline $\mathrm{LRF} \times(\mathrm{LCR} \times \mathrm{TR})-005$ & 265.43 & 11 & 2.57 & 35 & 11.94 & 11 & 47.22 & 19 & 1.87 & 18 & 3.31 & 29 & 123.000 & 17 \\
\hline CLEO $\times$ TR Rubidoux $-\mathrm{CO}$ & 151.20 & 43 & 2.45 & 36 & 12.99 & 1 & 47.93 & 12 & 1.49 & 31 & 2.72 & 1 & 124.000 & 18 \\
\hline Sunki Tropical mandarin & 291.21 & 6 & 2.72 & 30 & 11.52 & 27 & 48.09 & 9 & 1.97 & 12 & 3.73 & 41 & 125.000 & 19 \\
\hline CNPMF - 004 Rangpur lime & 302.69 & 4 & 3.19 & 20 & 11.23 & 38 & 47.36 & 17 & 2.15 & 4 & 3.81 & 44 & 127.000 & 20 \\
\hline HTR -069 & 215.20 & 26 & 4.33 & 2 & 10.58 & 46 & 46.05 & 26 & 1.85 & 19 & 3.07 & 12 & 131.000 & 21 \\
\hline HTR -051 & 244.33 & 17 & 3.48 & 11 & 11.58 & 22 & 45.21 & 35 & 1.78 & 21 & 3.28 & 27 & 133.000 & 22 \\
\hline CNPMF - 003 Rangpur lime & 239.38 & 20 & 3.33 & 19 & 10.81 & 45 & 47.10 & 21 & 2.08 & 6 & 3.26 & 25 & 136.000 & 23 \\
\hline $\mathrm{TSKC} \times(\mathrm{LCR} \times \mathrm{TR})-073$ & 253.95 & 13 & 3.32 & 16 & 11.67 & 18 & 45.45 & 32 & 1.67 & 25 & 3.49 & 37 & 141.000 & 24 \\
\hline $\mathrm{CLEO} \times \mathrm{CTCZ}-226$ & 230.46 & 23 & 3.06 & 28 & 11.77 & 14 & 45.40 & 33 & 1.52 & 28 & 3.22 & 23 & 149.000 & 25 \\
\hline Riverside citrandarin & 251.01 & 15 & 2.47 & 32 & 11.65 & 20 & 46.50 & 23 & 1.96 & 14 & 3.82 & 45 & 149.000 & 25 \\
\hline HTR - 116 & 246.13 & 16 & 4.07 & 8 & 11.32 & 35 & 43.80 & 45 & 1.46 & 35 & 3.06 & 11 & 150.000 & 27 \\
\hline LVK $\times$ LVA -009 & 215.68 & 25 & 2.72 & 33 & 12.39 & 4 & 44.21 & 42 & 1.77 & 22 & 3.27 & 26 & 152.000 & 28 \\
\hline TSKFL $\times$ CTC $25-010$ & 197.38 & 35 & 3.76 & 14 & 11.42 & 31 & 45.11 & 36 & 1.46 & 34 & 2.87 & 3 & 153.000 & 29 \\
\hline TSKFL $\times$ CTTR -022 & 161.02 & 41 & 2.88 & 31 & 11.56 & 25 & 47.55 & 15 & 1.19 & 42 & 2.87 & 2 & 156.000 & 30 \\
\hline TSKC $\times$ LHA -006 & 211.22 & 29 & 2.22 & 41 & 11.69 & 16 & 48.15 & 6 & 1.50 & 30 & 3.41 & 35 & 157.000 & 31 \\
\hline $\mathrm{TSKC} \times \mathrm{CTSW}-064$ & 205.34 & 33 & 3.92 & 18 & 11.45 & 30 & 45.92 & 28 & 1.35 & 37 & 3.08 & 14 & 160.000 & 32 \\
\hline TSKC $\times$ CTQT $1439-004$ & 208.50 & 31 & 2.09 & 42 & 11.55 & 26 & 48.51 & 4 & 1.70 & 24 & 3.34 & 33 & 160.000 & 32 \\
\hline TSK $\times$ CTTR -002 & 218.36 & 24 & 2.76 & 29 & 11.15 & 39 & 44.99 & 38 & 2.16 & 3 & 3.31 & 28 & 161.000 & 34 \\
\hline $\mathrm{LVK} \times \mathrm{LCR}-010$ & 242.26 & 18 & 3.33 & 24 & 10.99 & 41 & 44.98 & 39 & 1.77 & 23 & 3.20 & 20 & 165.000 & 35 \\
\hline TSKFL $\times$ CTTR -012 & 193.10 & 36 & 3.52 & 27 & 10.98 & 42 & 46.40 & 25 & 1.65 & 26 & 3.11 & 15 & 171.000 & 36 \\
\hline $\mathrm{TSKC} \times(\mathrm{LCR} \times \mathrm{TR})-018$ & 213.01 & 28 & 3.31 & 22 & 11.04 & 40 & 45.25 & 34 & 1.48 & 32 & 3.13 & 18 & 174.000 & 37 \\
\hline LCREEL $\times$ CTSW -001 & 149.48 & 44 & 2.51 & 34 & 12.07 & 7 & 44.82 & 40 & 1.36 & 36 & 3.22 & 22 & 183.000 & 38 \\
\hline TSKFL $\times$ CTTR -008 & 157.45 & 42 & 2.52 & 38 & 12.09 & 6 & 43.80 & 46 & 1.22 & 41 & 3.04 & 10 & 183.000 & 38 \\
\hline TSKC $\times$ LHA -011 & 172.43 & 39 & 1.95 & 39 & 11.98 & 10 & 45.00 & 37 & 1.58 & 27 & 3.43 & 36 & 188.000 & 40 \\
\hline TSKC $\times$ CTRK -001 & 145.00 & 45 & 2.00 & 44 & 12.06 & 8 & 47.03 & 22 & 1.07 & 45 & 3.32 & 30 & 194.000 & 41 \\
\hline TSKC $\times$ CTARG -036 & 171.80 & 40 & 2.26 & 40 & 11.47 & 29 & 45.58 & 31 & 1.29 & 38 & 3.21 & 21 & 199.000 & 42 \\
\hline Sunki $\times$ Alemow $-\mathrm{CO}$ & 124.79 & 46 & 2.62 & 37 & 11.34 & 34 & 45.65 & 30 & 1.04 & 46 & 3.01 & 9 & 202.000 & 43 \\
\hline Sunki Maravilha mandarin & 174.12 & 38 & 1.72 & 46 & 11.79 & 13 & 45.80 & 29 & 1.08 & 44 & 3.34 & 32 & 202.000 & 43 \\
\hline TSKC $\times$ CTSW -019 & 235.69 & 22 & 2.02 & 43 & 11.51 & 28 & 43.82 & 44 & 1.27 & 39 & 3.73 & 40 & 216.000 & 45 \\
\hline TSKC $\times$ CTARG -001 & 198.78 & 34 & 1.66 & 45 & 11.67 & 19 & 44.42 & 41 & 1.23 & 40 & 3.98 & 46 & 225.000 & 46 \\
\hline F & $* *$ & & $* *$ & & ** & & $* *$ & & $* *$ & & $* *$ & & & \\
\hline Coefficient of variation (\%) & 16.55 & & 20.37 & & 3.37 & & 4.13 & & 10.37 & & 6.32 & & & \\
\hline Mean & 227.28 & & 3.10 & & 11.61 & & 46.49 & & 1.68 & & 3.27 & & & \\
\hline
\end{tabular}

TSK, 'Sunki' mandarin [Citrus sunki (Hayata) hort. ex Tanaka]; TSKC, common 'Sunki' mandarin (C. sunki); TR, trifoliate orange [Poncirus trifoliata (L.) Raf.]; LCR, Rangpur lime (C. limonia Osbeck); CTSW, 'Swingle' citrumelo (C. paradisi Macfad. x P. trifoliata); HTR, trifoliate hybrid (P. trifoliata x sp.); LRF, 'Florida' rough lemon (C. jambhiri Lush.); CTQT, 'Thomasville' citrangequat [Fortunella margarita (Lour.) Swingle x 'Willits' citrange]; LHA, 'Hamlin' sweet orange [C. sinensis (L.) Osbeck]; CTCZ, 'Carrizo' citrange (C. sinensis x P. trifoliata); CTTR, 'Troyer' citrange (C. sinensis x P. trifoliata); LVK, 'Volkamer' lemon (C. volkameriana (Risso) V. Ten. \& Pasq.); LVA, 'Valência' sweet orange (C. sinensis); TSKFL, 'Florida Sunki' mandarin (C. sunki); CTC, citrange (C. sinensis x P. trifoliata); CLEO, 'Cleópatra' mandarin (C. reshni hort. ex Tanaka); CTARG, 'Argentina' citrange (C. sinensis x P. trifoliata); LCREEL, 'Santa Cruz' Rangpur lime (C. Limonia); CTRK, 'Rusk' citrange (C. sinensis x P. trifoliata); 'Alemow' (C. macrophylla Wester); English Palmira - CO: Sunki x English Palmira - CO is a selection of a USDA hybrid (C. sunki x P. trifoliata cv. English) introduced from the city of Palmira in Colombia; CNPMF, Embrapa Mandioca e Fruticultura. **Significant at $1 \%$ probability. The classification sum index was calculated based on the methodology described by Mulamba \& Mock (1978). 
Table 3. Original means of the accumulated production (AP), canopy production efficiency (EF), concentration of soluble solids (SS), juice yield (JC), drought-tolerance (DT) (visual scoring of leaf wilting), and tree height (TH) for the determination of the ranking index based on simple means $\left(\mathrm{I}_{\mathrm{RKi}}\right)$, for the classification of hybrid citrus rootstocks grafted with 'Valência' sweet orange in the north of São Paulo state, Brazil, 2009-2017.

\begin{tabular}{|c|c|c|c|c|c|c|c|c|}
\hline Rootstock & $\begin{array}{c}\text { AP } \\
\text { (kg per tree) }\end{array}$ & $\begin{array}{c}\mathrm{EF} \\
\left(\mathrm{kg} \mathrm{m}^{-3}\right)\end{array}$ & $\begin{array}{c}\mathrm{SS} \\
\left({ }^{\circ} \text { Brix }\right)\end{array}$ & $\begin{array}{c}\mathrm{JC} \\
(\%) \\
\end{array}$ & DT & $\begin{array}{l}\mathrm{TH} \\
(\mathrm{m}) \\
\end{array}$ & IRKi & Rank \\
\hline Indio citrandarin & 320.96 & 3.17 & 11.58 & 48.01 & 1.91 & 3.78 & 74.1719 & 1 \\
\hline Sunki $\times$ English Palmira $-\mathrm{CO}$ & 317.99 & 2.90 & 12.88 & 47.85 & 1.93 & 3.76 & 73.7003 & 2 \\
\hline San Diego citrandarin & 305.22 & 3.51 & 11.38 & 49.23 & 1.99 & 3.35 & 71.2656 & 3 \\
\hline CNPMF - 004 Rangpur lime & 302.69 & 3.19 & 11.23 & 47.36 & 2.15 & 3.81 & 70.4214 & 4 \\
\hline TSKC $\times$ CTSW -028 & 296.79 & 3.14 & 11.68 & 47.30 & 2.02 & 3.70 & 69.2661 & 5 \\
\hline Sunki Tropical mandarin & 291.21 & 2.72 & 11.52 & 48.09 & 1.97 & 3.73 & 68.1472 & 6 \\
\hline $\mathrm{HTR}-053$ & 287.26 & 3.87 & 11.27 & 45.94 & 2.05 & 3.34 & 67.2465 & 7 \\
\hline Cravo Santa Cruz Rangpur lime & 276.19 & 3.41 & 10.85 & 49.07 & 2.03 & 3.59 & 65.3446 & 8 \\
\hline TSKC $\times($ LCR $\times$ TR $)-017$ & 275.88 & 4.08 & 11.58 & 47.43 & 1.96 & 3.13 & 65.2682 & 9 \\
\hline $\mathrm{TSKC} \times \mathrm{CTSW}-041$ & 272.91 & 3.76 & 11.29 & 47.96 & 2.08 & 3.24 & 64.6683 & 10 \\
\hline $\mathrm{LRF} \times(\mathrm{LCR} \times \mathrm{TR})-005$ & 265.43 & 2.57 & 11.94 & 47.22 & 1.87 & 3.31 & 63.7902 & 11 \\
\hline TSKC $\times($ LCR $\times$ TR $)-073$ & 253.95 & 3.32 & 11.67 & 45.45 & 1.67 & 3.49 & 62.8789 & 14 \\
\hline TSKC $\times($ LCR $\times$ TR $)-059$ & 262.34 & 4.20 & 12.02 & 47.68 & 2.22 & 3.16 & 62.7394 & 12 \\
\hline $\mathrm{LCR} \times \mathrm{TR}-001$ & 252.05 & 4.14 & 10.94 & 47.15 & 2.23 & 3.00 & 60.4305 & 13 \\
\hline Riverside citrandarin & 251.01 & 2.47 & 11.65 & 46.50 & 1.96 & 3.82 & 59.8372 & 15 \\
\hline HTR - 116 & 246.13 & 4.07 & 11.32 & 43.80 & 1.46 & 3.06 & 58.6328 & 16 \\
\hline HTR - 051 & 244.33 & 3.48 & 11.58 & 45.21 & 1.78 & 3.28 & 58.4686 & 17 \\
\hline TSKC $\times($ LCR $\times$ TR $)-001$ & 241.65 & 3.76 & 11.75 & 46.48 & 1.51 & 2.97 & 58.1496 & 18 \\
\hline $\mathrm{LVK} \times \mathrm{LCR}-010$ & 242.26 & 3.33 & 10.99 & 44.98 & 1.77 & 3.20 & 57.8986 & 19 \\
\hline CNPMF - 003 Rangpur lime & 239.38 & 3.33 & 10.81 & 47.10 & 2.08 & 3.26 & 57.6750 & 20 \\
\hline $\mathrm{LVK} \times \mathrm{LCR}-038$ & 237.46 & 4.28 & 11.37 & 44.12 & 2.03 & 2.93 & 57.1117 & 21 \\
\hline TSKC $\times$ CTSW -019 & 235.69 & 2.02 & 11.51 & 43.82 & 1.27 & 3.73 & 56.1232 & 22 \\
\hline CLEO $\times$ CTCZ -226 & 230.46 & 3.06 & 11.77 & 45.40 & 1.52 & 3.22 & 55.6152 & 23 \\
\hline TSKC $\times$ CTQT $1434-010$ & 215.03 & 3.75 & 11.94 & 48.72 & 1.79 & 2.93 & 53.2449 & 24 \\
\hline TSK $\times$ CTTR -002 & 218.36 & 2.76 & 11.15 & 44.99 & 2.16 & 3.31 & 53.1050 & 25 \\
\hline HTR - 069 & 215.20 & 4.33 & 10.58 & 46.05 & 1.85 & 3.07 & 52.8033 & 26 \\
\hline $\mathrm{LVK} \times \mathrm{LVA}-009$ & 215.68 & 2.72 & 12.39 & 44.21 & 1.77 & 3.27 & 52.5535 & 27 \\
\hline TSK $\times$ TR Benecke $-\mathrm{CO}$ & 210.47 & 2.88 & 12.50 & 48.10 & 1.92 & 3.12 & 52.1767 & 28 \\
\hline TSKC $\times($ LCR $\times$ TR $)-018$ & 213.01 & 3.31 & 11.04 & 45.25 & 1.48 & 3.13 & 52.0340 & 29 \\
\hline TSKC $\times$ LHA -006 & 211.22 & 2.22 & 11.69 & 48.15 & 1.50 & 3.41 & 51.9952 & 30 \\
\hline TSKC $\times$ CTSW -033 & 208.00 & 3.62 & 11.59 & 48.27 & 1.46 & 3.07 & 51.6273 & 31 \\
\hline TSKC $\times$ CTQT $1439-004$ & 208.50 & 2.09 & 11.55 & 48.51 & 1.70 & 3.34 & 51.4974 & 32 \\
\hline TSKC $\times$ CTSW -064 & 205.34 & 3.92 & 11.45 & 45.92 & 1.35 & 3.08 & 50.7597 & 33 \\
\hline TSKFL $\times$ CTC $25-010$ & 197.38 & 3.76 & 11.42 & 45.11 & 1.46 & 2.87 & 49.0329 & 34 \\
\hline TSKC $\times$ CTARG -001 & 198.78 & 1.66 & 11.67 & 44.42 & 1.23 & 3.98 & 48.7740 & 35 \\
\hline TSKFL $\times$ CTTR -012 & 193.10 & 3.52 & 10.98 & 46.40 & 1.65 & 3.11 & 48.2929 & 36 \\
\hline TSKC $\times$ CTQT $1439-026$ & 188.45 & 3.54 & 12.23 & 48.14 & 1.19 & 2.87 & 47.7271 & 37 \\
\hline Sunki Maravilha mandarin & 174.12 & 1.72 & 11.79 & 45.80 & 1.08 & 3.34 & 44.0520 & 38 \\
\hline TSKC $\times$ LHA -011 & 172.43 & 1.95 & 11.98 & 45.00 & 1.58 & 3.43 & 43.7683 & 39 \\
\hline TSKC $\times$ CTARG -036 & 171.80 & 2.26 & 11.47 & 45.58 & 1.29 & 3.21 & 43.6578 & 40 \\
\hline TSKFL $\times$ CTTR -022 & 161.02 & 2.88 & 11.56 & 47.55 & 1.19 & 2.87 & 41.9213 & 41 \\
\hline TSKFL $\times$ CTTR -008 & 157.45 & 2.52 & 12.09 & 43.80 & 1.22 & 3.04 & 40.6525 & 42 \\
\hline CLEO $\times$ TR Rubidoux $-\mathrm{CO}$ & 151.20 & 2.45 & 12.99 & 47.93 & 1.49 & 2.72 & 40.2021 & 43 \\
\hline LCREEL $\times$ CTSW -001 & 149.48 & 2.51 & 12.07 & 44.82 & 1.36 & 3.22 & 39.2338 & 44 \\
\hline TSKC $\times$ CTRK -001 & 145.00 & 2.00 & 12.06 & 47.03 & 1.07 & 3.32 & 38.5086 & 45 \\
\hline Sunki $\times$ Alemow $-\mathrm{CO}$ & 124.79 & 2.62 & 11.34 & 45.65 & 1.04 & 3.01 & 34.2706 & 46 \\
\hline $\mathrm{F}$ & $* *$ & $* *$ & $* *$ & $* *$ & $* *$ & $* *$ & & \\
\hline Coefficient of variation (\%) & 16.55 & 20.37 & 3.37 & 4.13 & 10.37 & 6.32 & & \\
\hline Mean & 227.28 & 3.10 & 11.61 & 46.49 & 1.68 & 3.27 & & \\
\hline
\end{tabular}

TSK, 'Sunki' mandarin [Citrus sunki (Hayata) hort. ex Tanaka]; TSKC, common 'Sunki' mandarin (C. sunki); TR, trifoliate orange [Poncirus trifoliata (L.) Raf.]; LCR, Rangpur lime (C. limonia Osbeck); CTSW, 'Swingle' citrumelo (C. paradisi Macfad. x P. trifoliata); HTR, trifoliate hybrid (P. trifoliata x sp.); LRF, 'Florida' rough lemon (C. jambhiri Lush.); CTQT, 'Thomasville' citrangequat [Fortunella margarita (Lour.) Swingle x 'Willits' citrange]; LHA, 'Hamlin' sweet orange [C. sinensis (L.) Osbeck]; CTCZ, 'Carrizo' citrange (C. sinensis x P. trifoliata); CTTR, 'Troyer' citrange (C. sinensis x P. trifoliata); LVK, 'Volkamer' lemon (C. volkameriana (Risso) V. Ten. \& Pasq.); LVA, 'Valência' sweet orange (C. sinensis); TSKFL, 'Florida Sunki' mandarin (C. sunki); CTC, citrange (C. sinensis x P. trifoliata); CLEO, 'Cleópatra' mandarin (C. reshni hort. ex Tanaka); CTARG, 'Argentina' citrange (C. sinensis x P. trifoliata); LCREEL, 'Santa Cruz' Rangpur lime (C. Limonia); CTRK, 'Rusk' citrange (C. sinensis x P. trifoliata); 'Alemow' (C. macrophylla Wester); English Palmira - CO: Sunki x English Palmira - CO is a selection of a USDA hybrid (C. sunki x P. trifoliata cv. English) introduced from the city of Palmira in Colombia; CNPMF, Embrapa Mandioca e Fruticultura. $* *$ Significant at $1 \%$ probability. The ranking index $\left(\mathrm{I}_{\mathrm{RK}}\right)$ was adapted from Costa et al. $(2016)$ using simple means as $\mathrm{I}_{\mathrm{RKi}}=(\mathrm{AP} \times 0.2)+(\mathrm{EF} \times 0.2)+(\mathrm{SS} \times 0.15)+$ $(\mathrm{JC} \times 0.15)+(\mathrm{DT} \times 0.2)+((1 / \mathrm{PH}) \times 0.1)$. 
Table 4. Original and normalized (N) means of the accumulated production (AP), canopy production efficiency (EF), concentration of soluble solids (SS), juice yield (JC), drought-tolerance (DT) (visual scoring of leaf wilting), and tree height $(\mathrm{TH})$, for the determination of the ranking index based on linearly normalized means $\left(\mathrm{I}_{\mathrm{RKii}}\right)$, to classify hybrid citrus rootstocks grafted with 'Valência' sweet orange, in the north of São Paulo state, Brazil, 2009-2017.

\begin{tabular}{|c|c|c|c|c|c|c|c|c|c|c|c|c|c|c|}
\hline Rootstock & $\begin{array}{c}\text { AP } \\
\text { (kg per tree) }\end{array}$ & $\mathrm{N}_{\mathrm{AP}}$ & $\begin{array}{c}E F \\
-\left(\mathrm{kg} \mathrm{m}^{-3}\right) \\
\end{array}$ & $\mathrm{N}_{\mathrm{EF}}$ & $\begin{array}{c}\mathrm{SS} \\
\left({ }^{\circ} \text { Brix }\right)\end{array}$ & $\mathrm{N}_{\mathrm{SS}}$ & $\mathrm{JC}$ & $\begin{array}{l}\mathrm{N}_{\mathrm{JC}} \\
-(\%)-\end{array}$ & DT & $\mathrm{N}_{\mathrm{DT}}$ & $\begin{array}{l}\mathrm{TH} \\
(\mathrm{m})\end{array}$ & $\mathrm{N}_{(1 / \mathrm{TH})}$ & $\mathrm{I}_{\mathrm{RKii}}$ & Rank \\
\hline Sunki $\times$ English Palmira $-\mathrm{CO}$ & 317.99 & 0.98 & 2.90 & 0.46 & 12.88 & 0.96 & 47.85 & 0.75 & 1.93 & 0.75 & 3.76 & 0.77 & 0.824 & 1 \\
\hline TSKC × (LCR x TR $)-059$ & 262.34 & 0.70 & 4.20 & 0.95 & 12.02 & 0.60 & 47.68 & 0.72 & 2.22 & 0.99 & 3.16 & 1.80 & 0.782 & 2 \\
\hline Indio citrandarin & 320.96 & 1.00 & 3.17 & 0.56 & 11.58 & 0.42 & 48.01 & 0.77 & 1.91 & 0.73 & 3.78 & 0.75 & 0.771 & 3 \\
\hline San Diego citrandarin & 305.22 & 0.92 & 3.51 & 0.69 & 11.38 & 0.33 & 49.23 & 1.00 & 1.99 & 0.80 & 3.35 & 1.26 & 0.762 & 4 \\
\hline CNPMF - 004 Rangpur lime & 302.69 & 0.91 & 3.19 & 0.57 & 11.23 & 0.27 & 47.36 & 0.66 & 2.15 & 0.93 & 3.81 & 0.73 & 0.758 & 5 \\
\hline $\mathrm{TSKC} \times \mathrm{CTSW}-028$ & 296.79 & 0.88 & 3.14 & 0.55 & 11.68 & 0.46 & 47.30 & 0.64 & 2.02 & 0.83 & 3.70 & 0.81 & 0.740 & 6 \\
\hline Cravo Santa Cruz Rangpur lime & 276.19 & 0.77 & 3.41 & 0.66 & 10.85 & 0.11 & 49.07 & 0.97 & 2.03 & 0.84 & 3.59 & 0.91 & 0.725 & 7 \\
\hline Sunki Tropical mandarin & 291.21 & 0.85 & 2.72 & 0.39 & 11.52 & 0.39 & 48.09 & 0.79 & 1.97 & 0.78 & 3.73 & 0.78 & 0.709 & 8 \\
\hline $\mathrm{TSKC} \times \mathrm{CTSW}-041$ & 272.91 & 0.76 & 3.76 & 0.79 & 11.29 & 0.29 & 47.96 & 0.77 & 2.08 & 0.88 & 3.24 & 1.52 & 0.709 & 9 \\
\hline $\mathrm{TSKC} \times(\mathrm{LCR} \times \mathrm{TR})-017$ & 275.88 & 0.77 & 4.08 & 0.91 & 11.58 & 0.41 & 47.43 & 0.67 & 1.96 & 0.77 & 3.13 & 1.92 & 0.704 & 10 \\
\hline HTR -053 & 287.26 & 0.83 & 3.87 & 0.83 & 11.27 & 0.29 & 45.94 & 0.39 & 2.05 & 0.85 & 3.34 & 1.28 & 0.680 & 11 \\
\hline $\mathrm{LCR} \times \mathrm{TR}-001$ & 252.05 & 0.65 & 4.14 & 0.93 & 10.94 & 0.15 & 47.15 & 0.62 & 2.23 & 1.00 & 3.00 & 2.84 & 0.666 & 12 \\
\hline Riverside citrandarin & 251.01 & 0.64 & 2.47 & 0.30 & 11.65 & 0.44 & 46.50 & 0.50 & 1.96 & 0.77 & 3.82 & 0.72 & 0.623 & 13 \\
\hline TSKC $\times$ CTQT $1434-010$ & 215.03 & 0.46 & 3.75 & 0.78 & 11.94 & 0.56 & 48.72 & 0.91 & 1.79 & 0.63 & 2.93 & 3.73 & 0.621 & 14 \\
\hline TSK $\times$ TR Benecke $-\mathrm{CO}$ & 210.47 & 0.44 & 2.88 & 0.45 & 12.50 & 0.80 & 48.10 & 0.79 & 1.92 & 0.74 & 3.12 & 1.98 & 0.615 & 15 \\
\hline $\mathrm{LRF} \times(\mathrm{LCR} \times \mathrm{TR})-005$ & 265.43 & 0.72 & 2.57 & 0.34 & 11.94 & 0.57 & 47.22 & 0.63 & 1.87 & 0.70 & 3.31 & 1.35 & 0.604 & 16 \\
\hline CNPMF - 003 Rangpur lime & 239.38 & 0.58 & 3.33 & 0.62 & 10.81 & 0.10 & 47.10 & 0.61 & 2.08 & 0.87 & 3.26 & 1.47 & 0.590 & 17 \\
\hline TSKC $\times($ LCR $\times$ TR $)-073$ & 253.95 & 0.66 & 3.32 & 0.62 & 11.67 & 0.45 & 45.45 & 0.30 & 1.67 & 0.53 & 3.49 & 1.03 & 0.572 & 18 \\
\hline $\mathrm{LVK} \times \mathrm{LCR}-038$ & 237.46 & 0.57 & 4.28 & 0.98 & 11.37 & 0.33 & 44.12 & 0.06 & 2.03 & 0.83 & 2.93 & 3.83 & 0.562 & 19 \\
\hline HTR -051 & 244.33 & 0.61 & 3.48 & 0.68 & 11.58 & 0.42 & 45.21 & 0.26 & 1.78 & 0.63 & 3.28 & 1.43 & 0.555 & 20 \\
\hline HTR - 069 & 215.20 & 0.46 & 4.33 & 1.00 & 10.58 & 0.00 & 46.05 & 0.41 & 1.85 & 0.68 & 3.07 & 2.26 & 0.535 & 21 \\
\hline $\mathrm{TSKC} \times \mathrm{CTSW}-033$ & 208.00 & 0.42 & 3.62 & 0.73 & 11.59 & 0.42 & 48.27 & 0.82 & 1.46 & 0.36 & 3.07 & 2.26 & 0.534 & 22 \\
\hline TSKC $\times($ LCR $\times$ TR $)-001$ & 241.65 & 0.60 & 3.76 & 0.78 & 11.75 & 0.48 & 46.48 & 0.49 & 1.51 & 0.39 & 2.97 & 3.15 & 0.533 & 23 \\
\hline $\mathrm{TSK} \times \mathrm{CTTR}-002$ & 218.36 & 0.48 & 2.76 & 0.41 & 11.15 & 0.24 & 44.99 & 0.22 & 2.16 & 0.94 & 3.31 & 1.36 & 0.507 & 24 \\
\hline TSKC $\times$ CTQT $1439-004$ & 208.50 & 0.43 & 2.09 & 0.16 & 11.55 & 0.40 & 48.51 & 0.87 & 1.70 & 0.56 & 3.34 & 1.28 & 0.497 & 25 \\
\hline $\mathrm{LVK} \times \mathrm{LVA}-009$ & 215.68 & 0.46 & 2.72 & 0.40 & 12.39 & 0.75 & 44.21 & 0.08 & 1.77 & 0.61 & 3.27 & 1.46 & 0.487 & 26 \\
\hline $\mathrm{LVK} \times \mathrm{LCR}-010$ & 242.26 & 0.60 & 3.33 & 0.63 & 10.99 & 0.17 & 44.98 & 0.22 & 1.77 & 0.61 & 3.20 & 1.66 & 0.486 & 27 \\
\hline TSKC $\times$ LHA -006 & 211.22 & 0.44 & 2.22 & 0.21 & 11.69 & 0.46 & 48.15 & 0.80 & 1.50 & 0.39 & 3.41 & 1.15 & 0.485 & 28 \\
\hline $\mathrm{CLEO} \times \mathrm{CTCZ}-226$ & 230.46 & 0.54 & 3.06 & 0.52 & 11.77 & 0.50 & 45.40 & 0.30 & 1.52 & 0.41 & 3.22 & 1.58 & 0.475 & 29 \\
\hline TSKC $\times$ CTQT $1439-026$ & 188.45 & 0.32 & 3.54 & 0.70 & 12.23 & 0.69 & 48.14 & 0.80 & 1.19 & 0.13 & 2.87 & 5.22 & 0.473 & 30 \\
\hline HTR -116 & 246.13 & 0.62 & 4.07 & 0.90 & 11.32 & 0.31 & 43.80 & 0.00 & 1.46 & 0.35 & 3.06 & 2.33 & 0.464 & 31 \\
\hline $\mathrm{TSKC} \times \mathrm{CTSW}-064$ & 205.34 & 0.41 & 3.92 & 0.85 & 11.45 & 0.36 & 45.92 & 0.39 & 1.35 & 0.26 & 3.08 & 2.22 & 0.462 & 32 \\
\hline TSKFL $\times$ CTTR -012 & 193.10 & 0.35 & 3.52 & 0.70 & 10.98 & 0.17 & 46.40 & 0.48 & 1.65 & 0.51 & 3.11 & 2.03 & 0.457 & 33 \\
\hline CLEO $\times$ TR Rubidoux $-\mathrm{CO}$ & 151.20 & 0.13 & 2.45 & 0.29 & 12.99 & 1.00 & 47.93 & 0.76 & 1.49 & 0.38 & 2.72 & 357.65 & 0.426 & 34 \\
\hline TSKFL $\times$ CTC $25-010$ & 197.38 & 0.37 & 3.76 & 0.79 & 11.42 & 0.35 & 45.11 & 0.24 & 1.46 & 0.35 & 2.87 & 5.22 & 0.410 & 35 \\
\hline TSKC $\times($ LCR $\times$ TR $)-018$ & 213.01 & 0.45 & 3.31 & 0.62 & 11.04 & 0.19 & 45.25 & 0.27 & 1.48 & 0.37 & 3.13 & 1.92 & 0.408 & 36 \\
\hline TSKC $\times$ LHA -011 & 172.43 & 0.24 & 1.95 & 0.11 & 11.98 & 0.58 & 45.00 & 0.22 & 1.58 & 0.46 & 3.43 & 1.11 & 0.372 & 37 \\
\hline TSKC × CTSW - 019 & 235.69 & 0.57 & 2.02 & 0.13 & 11.51 & 0.39 & 43.82 & 0.00 & 1.27 & 0.20 & 3.73 & 0.79 & 0.365 & 38 \\
\hline TSKC $\times$ CTARG -001 & 198.78 & 0.38 & 1.66 & 0.00 & 11.67 & 0.45 & 44.42 & 0.12 & 1.23 & 0.16 & 3.98 & 0.63 & 0.351 & 39 \\
\hline TSKFL $\times$ CTTR -022 & 161.02 & 0.18 & 2.88 & 0.46 & 11.56 & 0.41 & 47.55 & 0.69 & 1.19 & 0.13 & 2.87 & 5.42 & 0.338 & 40 \\
\hline LCREEL $\times$ CTSW -001 & 149.48 & 0.13 & 2.51 & 0.32 & 12.07 & 0.62 & 44.82 & 0.19 & 1.36 & 0.27 & 3.22 & 1.60 & 0.326 & 41 \\
\hline TSKC $\times$ CTRK -001 & 145.00 & 0.10 & 2.00 & 0.13 & 12.06 & 0.61 & 47.03 & 0.60 & 1.07 & 0.03 & 3.32 & 1.33 & 0.308 & 42 \\
\hline TSKC $\times$ CTARG -036 & 171.80 & 0.24 & 2.26 & 0.22 & 11.47 & 0.37 & 45.58 & 0.33 & 1.29 & 0.22 & 3.21 & 1.64 & 0.301 & 43 \\
\hline Sunki Maravilha mandarin & 174.12 & 0.25 & 1.72 & 0.02 & 11.79 & 0.50 & 45.80 & 0.37 & 1.08 & 0.03 & 3.34 & 1.28 & 0.270 & 44 \\
\hline TSKFL $\times$ CTTR -008 & 157.45 & 0.17 & 2.52 & 0.32 & 12.09 & 0.63 & 43.80 & 0.00 & 1.22 & 0.15 & 3.04 & 2.49 & 0.261 & 45 \\
\hline Sunki $\times$ Alemow $-\mathrm{CO}$ & 124.79 & 0.00 & 2.62 & 0.36 & 11.34 & 0.31 & 45.65 & 0.34 & 1.04 & 0.00 & 3.01 & 2.73 & 0.207 & 46 \\
\hline Mean & 227.28 & & 3.10 & & 11.61 & & 46.49 & & 1.68 & & 3.27 & & & \\
\hline Maximum & 320.96 & & 4.33 & & 12.99 & & 49.23 & & 2.23 & & 3.98 & & & \\
\hline Minimum & 124.79 & & 1.66 & & 10.58 & & 43.80 & & 1.04 & & 2.72 & & & \\
\hline $\mathrm{F}$ & $* *$ & & $* *$ & & $* *$ & & $* *$ & & $* *$ & & $* *$ & & & \\
\hline Coefficient of variation (\%) & 16.55 & & 20.37 & & 3.37 & & 4.13 & & 10.37 & & 6.32 & & & \\
\hline
\end{tabular}

TSK, 'Sunki' mandarin [Citrus sunki (Hayata) hort. ex Tanaka]; TSKC, common 'Sunki' mandarin (C. sunki); TR, trifoliate orange [Poncirus trifoliata (L.) Raf.]; LCR, Rangpur lime (C. limonia Osbeck); CTSW, 'Swingle' citrumelo (C. paradisi Macfad. x P. trifoliata); HTR, trifoliate hybrid (P. trifoliata x sp.); LRF, 'Florida' rough lemon (C. jambhiri Lush.); CTQT, 'Thomasville' citrangequat [Fortunella margarita (Lour.) Swingle x 'Willits' citrange]; LHA, 'Hamlin' sweet orange [C. sinensis (L.) Osbeck]; CTCZ, 'Carrizo' citrange (C. sinensis x P. trifoliata); CTTR, 'Troyer' citrange (C. sinensis x P. trifoliata); LVK, 'Volkamer' lemon (C. volkameriana (Risso) V. Ten. \& Pasq.); LVA, 'Valência' sweet orange (C. sinensis); TSKFL, 'Florida Sunki' mandarin (C. sunki); CTC, citrange (C. sinensis x P. trifoliata); CLEO, 'Cleópatra' mandarin (C. reshni hort. ex Tanaka); CTARG, 'Argentina' citrange (C. sinensis x P. trifoliata); LCREEL, 'Santa Cruz' Rangpur lime (C. Limonia); CTRK, 'Rusk' citrange (C. sinensis x P. trifoliata); 'Alemow' (C. macrophylla Wester); English Palmira - CO: Sunki x English Palmira - CO is a selection of a USDA hybrid (C. sunki x P. trifoliata cv. English) introduced from the city of Palmira in Colombia; CNPMF, Embrapa Mandioca e Fruticultura. **Significant at $1 \%$ probability. The ranking index $\left(\mathrm{I}_{\mathrm{RKii}}\right)$ was adapted from Costa et al. (2016), with data subjected to linear normalization. 
Table 5. Original means of the accumulated production (AP), canopy production efficiency (EF), concentration of soluble solids (SS), juice yield (JC), drought-tolerance (DT) visual scoring of leaf wilting, and tree height (TH) for the determination of the index $\left(\mathrm{D}_{\mathrm{iI}}\right)$ based on the Euclidean distances $\left(\mathrm{d}_{\mathrm{ij}}\right)$ between genotype and ideotype, to classify hybrid citrus rootstocks grafted with 'Valência' sweet orange, in the north of São Paulo state, Brazil, 2009-2017.

\begin{tabular}{|c|c|c|c|c|c|c|c|c|c|c|c|c|c|c|}
\hline Rootstock & $\begin{array}{c}\text { AP } \\
\text { (kg per tree) }\end{array}$ & $\mathrm{d}_{\mathrm{ij}}$ & $\begin{array}{c}\mathrm{EF} \\
\left(\mathrm{kg} \mathrm{m}^{-3}\right)\end{array}$ & $\mathrm{d}_{\mathrm{ij}}$ & $\begin{array}{c}\text { SS } \\
\left({ }^{\circ} \text { Brix }\right)\end{array}$ & $d_{i j}$ & $\begin{array}{l}\mathrm{JC} \\
(\%) \\
\end{array}$ & $\mathrm{d}_{\mathrm{ij}}$ & DT & $\mathrm{d}_{\mathrm{ij}}$ & $\begin{array}{l}\mathrm{TH} \\
(\mathrm{m})\end{array}$ & $d_{i j}$ & $D_{\text {il }}$ & Rank \\
\hline TSKC $\times$ CTARG -001 & 198.78 & -1.04 & 1.66 & -3.19 & 11.67 & 1.33 & 44.42 & -3.60 & 1.23 & -2.23 & 3.98 & -101.59 & 101.7449 & 1 \\
\hline Riverside citrandarin & 251.01 & 0.02 & 2.47 & -2.08 & 11.65 & 1.29 & 46.50 & -2.26 & 1.96 & -0.12 & 3.82 & -101.20 & 101.2554 & 2 \\
\hline CNPMF - 004 Rangpur lime & 302.69 & 1.07 & 3.19 & -1.11 & 11.23 & 0.46 & 47.36 & -1.70 & 2.15 & 0.43 & 3.81 & -101.17 & 101.2002 & 3 \\
\hline Sunki $x$ English Palmira - CO & 317.99 & 1.39 & 2.90 & -1.51 & 12.88 & 3.75 & 47.85 & -1.38 & 1.93 & -0.21 & 3.76 & -101.05 & 101.1464 & 4 \\
\hline Indio citrandarin & 320.96 & 1.45 & 3.17 & -1.14 & 11.58 & 1.15 & 48.01 & -1.29 & 1.91 & -0.25 & 3.78 & -101.10 & 101.1326 & 5 \\
\hline $\mathrm{TSKC} \times \mathrm{CTSW}-019$ & 235.69 & -0.29 & 2.02 & -2.71 & 11.51 & 1.02 & 43.82 & -3.98 & 1.27 & -2.10 & 3.73 & -100.97 & 101.1118 & 6 \\
\hline Sunki Tropical mandarin & 291.21 & 0.84 & 2.72 & -1.75 & 11.52 & 1.04 & 48.09 & -1.23 & 1.97 & -0.10 & 3.73 & -100.98 & 101.0159 & 7 \\
\hline TSKC $\times$ CTSW -028 & 296.79 & 0.95 & 3.14 & -1.17 & 11.68 & 1.36 & 47.30 & -1.74 & 2.02 & 0.07 & 3.70 & -100.90 & 100.9306 & 8 \\
\hline Cravo Santa Cruz Rangpur lime & 276.19 & 0.53 & 3.41 & -0.80 & 10.85 & -0.29 & 49.07 & -0.60 & 2.03 & 0.10 & 3.59 & -100.60 & 100.6086 & 9 \\
\hline TSKC $\times($ TR $\times$ LCR $)-073$ & 253.95 & 0.08 & 3.32 & -0.93 & 11.67 & 1.33 & 45.45 & -2.93 & 1.67 & -0.97 & 3.49 & -100.31 & 100.3680 & 10 \\
\hline TSKC $\times$ LHA -011 & 172.43 & -1.58 & 1.95 & -2.80 & 11.98 & 1.96 & 45.00 & -3.22 & 1.58 & -1.22 & 3.43 & -100.12 & 100.2491 & 11 \\
\hline TSKC $\times$ LHA -006 & 211.22 & -0.79 & 2.22 & -2.43 & 11.69 & 1.38 & 48.15 & -1.19 & 1.50 & -1.44 & 3.41 & -100.05 & 100.1086 & 12 \\
\hline Sunki Maravilha mandarin & 174.12 & -1.55 & 1.72 & -3.12 & 11.79 & 1.58 & 45.80 & -2.71 & 1.08 & -2.67 & 3.34 & -99.81 & 99.9602 & 13 \\
\hline TSKC × CTRK -001 & 145.00 & -2.14 & 2.00 & -2.73 & 12.06 & 2.11 & 47.03 & -1.91 & 1.07 & -2.68 & 3.32 & -99.74 & 99.8776 & 14 \\
\hline TSKC $\times$ CTQT $1439-004$ & 208.50 & -0.85 & 2.09 & -2.61 & 11.55 & 1.09 & 48.51 & -0.96 & 1.70 & -0.86 & 3.34 & -99.81 & 99.8668 & 15 \\
\hline San Diego citrandarin & 305.22 & 1.13 & 3.51 & -0.66 & 11.38 & 0.75 & 49.23 & -0.50 & 1.99 & -0.02 & 3.35 & -99.85 & 99.8 & 16 \\
\hline HTR -053 & 287.26 & 0.76 & 3.87 & -0.18 & 11.27 & 0.54 & 45.94 & -2.62 & 2.05 & 0.14 & 3.34 & -99.81 & 99.8536 & 17 \\
\hline TSK $\times$ CTTR -002 & 218.36 & -0.65 & 2.76 & -1.69 & 11.15 & 0.29 & 44.99 & -3.23 & 2.16 & 0.45 & 3.31 & -99.70 & 99.7733 & 18 \\
\hline $\mathrm{LRF} \times(\mathrm{LCR} \times \mathrm{TR})-005$ & 265.43 & 0.31 & 2.57 & -1.95 & 11.94 & 1.88 & 47.22 & -1.79 & 1.87 & -0.39 & 3.31 & -99.71 & 99.7665 & 19 \\
\hline $\mathrm{LVK} \times \mathrm{LVA}-009$ & 215.68 & -0.70 & 2.72 & -1.75 & 12.39 & 2.76 & 44.21 & -3.73 & 1.77 & -0.67 & 3.27 & -99.57 & 99.6933 & 20 \\
\hline HTR -051 & 244.33 & -0.12 & 3.48 & -0.72 & 11.58 & 1.16 & 45.21 & -3.09 & 1.78 & -0.63 & 3.28 & -99.61 & 99.6675 & 21 \\
\hline CNPMF - 003 Rangpur lime & 239.38 & -0.22 & 3.33 & -0.92 & 10.81 & -0.37 & 47.10 & -1.87 & 2.08 & 0.23 & 3.26 & -99.55 & 99.5737 & 22 \\
\hline LCREEL $\times$ CTSW -001 & 149.48 & -2.05 & 2.51 & -2.04 & 12.07 & 2.14 & 44.82 & -3.34 & 1.36 & -1.86 & 3.22 & -99.39 & 99.5325 & 23 \\
\hline TSKC $\times$ CTSW -041 & 272.91 & 0.47 & 3.76 & -0.32 & 11.29 & 0.57 & 47.96 & -1.32 & 2.08 & 0.24 & 3.24 & -99.49 & 99.50 & 24 \\
\hline CLEO × CTCZ - 226 & 230.46 & -0.40 & 3.06 & -1.29 & 11.77 & 1.54 & 45.40 & -2.97 & 1.52 & -1.39 & 3.22 & 99.41 & 888 & 25 \\
\hline TSKC $\times$ CTARG -036 & 171.80 & -1.59 & 2.26 & -2.38 & 11.47 & 0.93 & 45.58 & -2.85 & 1.29 & -2.05 & 3.21 & -99.35 & 99.4 & 26 \\
\hline $\mathrm{LVK} \times \mathrm{LCR}-010$ & 242.26 & -0.16 & 3.33 & -0.91 & 10.99 & -0.01 & 44.98 & -3.24 & 1.77 & -0.68 & 3.20 & -99.33 & 99.3935 & 27 \\
\hline TSKC $\times($ LCR $\times$ TR $)-059$ & 262.34 & 0.25 & 4.20 & 0.27 & 12.02 & 2.03 & 47.68 & -1.49 & 2.22 & 0.65 & 3.16 & -99.19 & 99.2269 & 28 \\
\hline TSKC $\times($ LCR $\times$ TR $)-018$ & 213.01 & -0.75 & 3.31 & -0.95 & 11.04 & 0.07 & 45.25 & -3.06 & 1.48 & -1.51 & 3.13 & -99.09 & 99.1546 & 29 \\
\hline TSK × TR Benecke $-\mathrm{CO}$ & 210.47 & -0.81 & 2.88 & -1.53 & 12.50 & 2.98 & 48.10 & -1.22 & 1.92 & -0.23 & 3.12 & -99.05 & 99.1144 & 30 \\
\hline $\mathrm{TSKC} \times(\mathrm{LCR} \times \mathrm{TR})-017$ & 275.88 & 0.53 & 4.08 & 0.11 & 11.58 & 1.15 & 47.43 & -1.65 & 1.96 & -0.12 & 3.13 & -99.09 & 99.1105 & 31 \\
\hline TSKFL $\times$ CTTR -012 & 193.10 & -1.16 & 3.52 & -0.65 & 10.98 & -0.04 & 46.40 & -2.32 & 1.65 & -1.02 & 3.11 & -99.00 & 99.0457 & 32 \\
\hline TSKC $\times$ CTSW -064 & 205.34 & -0.91 & 3.92 & -0.11 & 11.45 & 0.90 & 45.92 & -2.63 & 1.35 & -1.88 & 3.08 & -98.88 & 98.9368 & 33 \\
\hline HTR -116 & 246.13 & -0.08 & 4.07 & 0.10 & 11.32 & 0.64 & 43.80 & -3.99 & 1.46 & -1.56 & 3.06 & -98.81 & 98.9054 & 34 \\
\hline HTR -069 & 215.20 & -0.71 & 4.33 & 0.45 & 10.58 & -0.84 & 46.05 & -2.55 & 1.85 & -0.42 & 3.07 & 98.85 & 948 & 35 \\
\hline TSK & 157 & -1.89 & 2.5 & -2.03 & 12.09 & 2.16 & 43.80 & -4.00 & 1.22 & -2.27 & 3.04 & & & 36 \\
\hline TSKC $\times$ & 208.00 & -0.86 & 3.62 & -0.52 & 11.59 & 1.18 & 48.27 & -1.12 & 1.46 & -1.55 & 3.07 & -98.85 & 98.8845 & 37 \\
\hline Sunki $\times$ Alemow $-\mathrm{CO}$ & 124.79 & -2.55 & 2.62 & -1.89 & 11.34 & 0.67 & 45.65 & -2.80 & 1.04 & -2.79 & 3.01 & -98.61 & 98.7422 & 38 \\
\hline $\mathrm{LCR} \times \mathrm{TR}-001$ & 252.05 & 0.04 & 4.14 & 0.19 & 10.94 & -0.11 & 47.15 & -1.84 & 2.23 & 0.67 & 3.00 & -98.56 & 98.5839 & 39 \\
\hline $\mathrm{TSKC} \times(\mathrm{LCR} \times \mathrm{TR})-001$ & 241.65 & -0.17 & 3.76 & -0.33 & 11.75 & 1.49 & 46.48 & -2.27 & 1.51 & -1.43 & 2.97 & -98.45 & 98.4975 & 40 \\
\hline $\mathrm{LVK} \times \mathrm{LCR}-038$ & 237.46 & -0.26 & 4.28 & 0.39 & 11.37 & 0.73 & 44.12 & -3.79 & 2.03 & 0.09 & 2.93 & -98.26 & 98.3372 & 41 \\
\hline TSKC $\times$ CTQT $1434-010$ & 215.03 & -0.71 & 3.75 & -0.34 & 11.94 & 1.87 & 48.72 & -0.83 & 1.79 & -0.62 & 2.93 & -98.28 & 98.3106 & 42 \\
\hline TSKC $\times$ CTQT $1439-026$ & 188.45 & -1.26 & 3.54 & -0.63 & 12.23 & 2.45 & 48.14 & -1.20 & 1.19 & -2.35 & 2.87 & -98.02 & 98.0922 & 43 \\
\hline TSKFL $\times$ CTC $25-010$ & 197.38 & -1.07 & 3.76 & -0.33 & 11.42 & 0.83 & 45.11 & -3.15 & 1.46 & -1.56 & 2.87 & -98.02 & 98.0892 & 44 \\
\hline TSKFL × CTTR -022 & 161.02 & -1.81 & 2.88 & -1.53 & 11.56 & 1.11 & 47.55 & -1.58 & 1.19 & -2.34 & 2.87 & -97.99 & 98.0667 & 45 \\
\hline CLEO $\times$ TR Rubidoux - CO & 151.20 & -2.01 & 2.45 & -2.12 & 12.99 & 3.96 & 47.93 & -1.33 & 1.49 & -1.48 & 2.72 & -97.31 & 97.4518 & 46 \\
\hline $\mathrm{Xij}=$ mean & 227.28 & & 3.10 & & 11.61 & & 46.49 & & 1.68 & & 3.27 & & & \\
\hline Variance & 2405.23 & & 0.54 & & 0.25 & & 2.41 & & 0.12 & & 0.09 & & & \\
\hline Standard deviation & 49.04 & & 0.73 & & 0.50 & & 1.55 & & 0.35 & & 0.30 & & & \\
\hline Ideotype & 250.00 & & 4.00 & & 11.00 & & 50.00 & & 2.00 & & 3.00 & & & \\
\hline $\mathrm{F}$ & $* *$ & & $* *$ & & $* *$ & & $* *$ & & $* *$ & & $* *$ & & & \\
\hline Coefficient of variation (\%) & 16.55 & & 20.37 & & 3.37 & & 4.13 & & 10.37 & & 6.32 & & & \\
\hline
\end{tabular}

TSK, 'Sunki' mandarin [Citrus sunki (Hayata) hort. ex Tanaka]; TSKC, common 'Sunki' mandarin (C. sunki); TR, trifoliate orange [Poncirus trifoliata (L.) Raf.]; LCR, Rangpur lime (C. limonia Osbeck); CTSW, 'Swingle' citrumelo (C. paradisi Macfad. x P. trifoliata); HTR, trifoliate hybrid ( $P$. trifoliata x sp.); LRF, 'Florida' rough lemon (C. jambhiri Lush.); CTQT, 'Thomasville' citrangequat [Fortunella margarita (Lour.) Swingle x 'Willits' citrange]; LHA, 'Hamlin' sweet orange [C. sinensis (L.) Osbeck]; CTCZ, 'Carrizo' citrange (C. sinensis x P. trifoliata); CTTR, 'Troyer' citrange (C. sinensis x P. trifoliata); LVK, 'Volkamer' lemon (C. volkameriana (Risso) V. Ten. \& Pasq.); LVA, 'Valência' sweet orange (C. sinensis); TSKFL, 'Florida Sunki' mandarin (C. sunki); CTC, citrange (C. sinensis x P. trifoliata); CLEO, 'Cleópatra' mandarin (C. reshni hort. ex Tanaka); CTARG, 'Argentina' citrange (C. sinensis x P. trifoliata); LCREEL, 'Santa Cruz' Rangpur lime (C. Limonia); CTRK, 'Rusk' citrange (C. sinensis x P. trifoliata); 'Alemow' (C. macrophylla Wester); English Palmira - CO: Sunki x English Palmira - CO is a selection of a USDA hybrid (C. sunki x P. trifoliata cv. English) introduced from the city of Palmira in Colombia; CNPMF, Embrapa Mandioca e Fruticultura. ${ }^{*}$ Significant at $1 \%$ probability. The genotype-ideotype index was calculated based on the methodology described by Schwarzbach (1972). 
$\times$ 'English Palmira' $-\mathrm{CO}$, and TSKC $\times($ LCR $\times$ TR $)-$ 059 ranked very often among the best genotypes for all indices. CNPMF - 004 Rangpur lime also surpassed the standard 'Cravo Santa Cruz' Rangpur lime. These results confirm the initial good performance of these hybrids, which are promising rootstocks for 'Valência' orange in rainfed cultivation, in São Paulo, Brazil, as reported by Ramos et al. (2015).

The Spearman correlation indicated a high similarity among the nonparametric indices, except for $D_{\text {iI }}$ (Table 6). The correlations were highly significant for $\mathrm{I}_{\mathrm{MMi}} \times \mathrm{I}_{\mathrm{RKi}}, \mathrm{I}_{\mathrm{MMi}} \times \mathrm{I}_{\text {RKii }}, \mathrm{I}_{\mathrm{MMi}} \times \mathrm{I}_{\mathrm{Ei}}, \mathrm{I}_{\mathrm{RKi}} \times \mathrm{I}_{\mathrm{RKii}}, \mathrm{I}_{\mathrm{RKi}} \times \mathrm{I}_{\mathrm{Ei}}, \mathrm{I}_{\mathrm{RKi}}$ $x D_{\mathrm{iI}}, \mathrm{I}_{\mathrm{RKii}} \times \mathrm{I}_{\mathrm{Ei}}$, and $\mathrm{I}_{\mathrm{RKii}} \times \mathrm{D}_{\mathrm{il}}$. The multiplicative index, as well as the classification sum and the genotypeideotype distance indices have been shown also to correlate well for other crops and to provide selection gains in hybrid populations (Lessa et al., 2010, 2017; Almeida et al., 2014). However, in the present work, the genotype-ideotype index was the most divergent because it prioritized relatively productive yet less efficient hybrid rootstocks. Nevertheless, $D_{\text {iI }}$ indicated several hybrids ranking above the ideotype for most variables; hence, it still helped out with the selection of promising genotypes.

The selection of new citrus rootstocks is a lifelong challenge for horticulturists, since dozens of traits should be observed, considering all influences that come from climate, soil type, tree management, scion/ rootstock combinations, occurrence of pests, and economic aspects (Castle, 2010). The highest yield

Table 6. Spearman's correlation among five nonparametric indices, using the variables accumulated fruit production, canopy production efficiency, concentration of soluble solids, juice yield, drought-tolerance (visual scoring of leaf wilting), and tree height of 'Valência' sweet orange grafted on 46 hybrid citrus rootstocks, in the north of São Paulo state, Brazil.

\begin{tabular}{lccccc}
\hline & $\mathrm{I}_{\mathrm{MMi}}$ & $\mathrm{I}_{\mathrm{RKi}}$ & $\mathrm{I}_{\mathrm{RKii}}$ & $\mathrm{I}_{\mathrm{Ei}}$ & $\mathrm{D}_{\mathrm{iI}}$ \\
\hline $\mathrm{I}_{\mathrm{MMi}}$ & - & & & & \\
$\mathrm{I}_{\mathrm{RKi}}$ & $0.68^{* *}$ & - & & & \\
$\mathrm{I}_{\mathrm{RKii}}$ & $0.85^{* *}$ & $0.91^{* *}$ & - & & \\
$\mathrm{I}_{\mathrm{Ei}}$ & $0.86^{* *}$ & $0.53^{* *}$ & $0.69^{* *}$ & - & \\
$\mathrm{D}_{\mathrm{il}}$ & $0.16^{\mathrm{ns}}$ & $0.43^{* *}$ & $0.30^{*}$ & $0.16^{\text {ns }}$ & - \\
\hline
\end{tabular}

$\mathrm{I}_{\mathrm{MMi}}$, classification sum index (Mulamba \& Mock, 1978); $\mathrm{I}_{\mathrm{RK}}$, ranking index using simple means (Costa et al., 2016); $I_{\text {RKii, }}$, ranking index using linearly normalized means; $\mathrm{I}_{\mathrm{Ei}}$, multiplicative index (Elston, 1963); $\mathrm{D}_{\mathrm{il}}$, genotype-ideotype distance index (Schwarzbach, 1972). *, **Significant

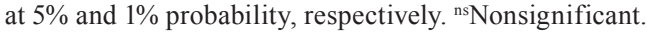

of fruits that meet the industrial standards is still the most important criterion, although reduced tree size is increasing in importance (Bowman et al., 2016). Classic univariate analyses such as LSD and other mean comparison and grouping tests are usually used to support decisions; however, for citrus, the analyses are still difficult due to their large genetic variability within several attributes.

The association of selection indices with other statistical tools was recommended, for more robust selection of genotypes (Ferreira et al., 2005). This is particularly important, since the indices discriminate, or should discriminate, the best genotypes, despite the challenges to attain a perfect correspondence because a single genotype will rarely satisfy all traits of interest (Lessa et al., 2010). Nonetheless, citrus breeders should choose the variables and indices that best fit the breeding objectives or economic interests, aiming at a more accurate selection gain, and further criteria can be applied, such as responses to diseases in the long term.

The five nonparametric indices were efficient to sort hybrid citrus rootstocks, even though each one prioritized different variables for selection. Moreover, the high correlations among indices showed that most of them can be similarly used to assist the selection of rootstocks with good overall performance. The ranking indices provided a more coherent classification of hybrids, particularly the $\mathrm{I}_{\mathrm{RKii}}$, which made it possible the ranking of the most productive genotypes with fair drought-tolerance and high-quality fruit for processing.

\section{Conclusions}

1. The use of nonparametric indices is suitable to assist the breeding programs for the selection of hybrid citrus rootstocks.

2. The ranking index based on the linear normalization of means allows of a more reliable classification of hybrid citrus rootstocks, since it highlights those presenting the greatest accumulated fruit production, in addition to good drought-tolerance, and efficient production of high-quality fruit to obtain juice.

\section{Acknowledgments}

To Coordenação de Aperfeiçoamento de Pessoal de Ensino Superior (Capes, Finance Code 001), for the doctoral scholarship granted to the first author; 
to Embrapa Mandioca e Fruticultura (CNPMF), for providing the plant materials and for financial support (Process 20.18.01.007.00.00); to Citrosuco S/A Agroindústria and to Fazenda Muriti, for technical support and permission to use the experimental area; and to Fundação Coopercitrus Credicitrus (FCC), for technical support.

\section{References}

ALMEIDA, L.M.; VIANA, A.P.; AMARAL JÚNIOR, A.T. do; CARNEIRO JÚNIOR, J. de B. Breeding full-sib families of sugar cane using selection index. Ciência Rural, v.44, p.605-611, 2014. DOI: https://doi.org/10.1590/S0103-84782014000400005.

AULER, P.A.M.; FIORI-TUTIDA, A.C.G.; TAZIMA, Z.H. Comportamento da laranjeira 'Valência' sobre seis porta-enxertos no noroeste do Paraná. Revista Brasileira de Fruticultura, v.30, p.229-234, 2008. DOI: https://doi.org/10.1590/S010029452008000100042.

BASTOS, D.C.; FERREIRA, E.A.; PASSOS, O.S.; SÁ, J.F. de; ATAÍDE, E.M.; CALGARO, M. Cultivares copa e porta-enxertos para a citricultura brasileira. Informe Agropecuário, v.35, p.3645, 2014.

BOWMAN, K.D.; FAULKNER, L.; KESINGER, M. New citrus rootstocks released by USDA 2001-2010: field performance and nursery characteristics. HortScience, v.51, p.1208-1214, 2016. DOI: https://doi.org/10.21273/HORTSCI10970-16.

CANTUARIAS-AVILÉS, T.; MOURÃO FILHO, F. de A.A.; STUCHI, E.S.; SILVA, S.R. da; ESPINOZA-NÚÑEZ, E. Horticultural performance of 'Folha Murcha' sweet orange onto twelve rootstocks. Scientia Horticulturae, v.129, p.259-265, 2011. DOI: https://doi.org/10.1016/j.scienta.2011.03.039.

CAPUTO, M.M.; MOURÃO FILHO, F. de A.A.; SILVA, S.R. da; BREMER NETO, H.; COUTO, H.T.Z. do; STUCHI, E.S. Seleção de cultivares de laranja doce de maturação precoce por índices de desempenho. Pesquisa Agropecuária Brasileira, v.47, p.1669-1672, 2012. DOI: https://doi.org/10.1590/S0100204 X2012001100015.

CASTLE, W.S. A career perspective on citrus rootstocks, their development, and commercialization. HortScience, v.45, p.11-15, 2010. DOI: https://doi.org/10.21273/HORTSCI.45.1.11.

CEPAGRI. Centro de Pesquisas Meteorológicas e Climáticas Aplicadas à Agricultura. Clima dos municípios paulistas: Colômbia. 2018. Available at: <https://www.cpa.unicamp.br>. Accessed on: Sept. 102018.

COSTA NETO, P.L.O. Estatística. São Paulo: Edgard Blücher, v.2, p.266, 2002.

COSTA, D.P.; STUCHI, E.S.; GIRARDI, E.A.; RAMOS, Y.C.; FADEL, A.L.; MALDONADO JUNIOR, W.; GESTEIRA, A. da S.; PASSOS, O.S.; SOARES FILHO, W. dos S. Potential rootstocks for Valencia sweet orange under rain-fed cultivation in the North of São Paulo, Brazil. Citrus Research \& Technology, v.37, p.26-36, 2016. DOI: https://doi.org/10.4322/crt.ICC038.
DOVALE, J.C.; FRITSCHE-NETO, R.; SILVA, P.S.L. e. Índice de seleção para cultivares de milho com dupla aptidão: minimilho e milho verde. Bragantia, v.70, p.781-787, 2011. DOI: https://doi.org/10.1590/S0006-87052011000400008.

ELSTON, R.C. A weight free index for the purpose of ranking or selection with respect to several traits at a time. Biometrics, v.19, p.85-97, 1963. DOI: https://doi.org/10.2307/2527573.

FERREIRA, A.; CECON, P.R.; CRUZ, C.D.; FERRÃO, R.G.; SILVA, M.F. da; FONSECA, A.F.A. da; FERRÃO, M.A.G. Seleção simultânea de Coffea canephora por meio da combinação de análise de fatores e índices de seleção. Pesquisa Agropecuária Brasileira, v.40, p.1189-1195, 2005. DOI: https://doi.org/10.1590/ S0100-204X2005001200005.

GARCIA, A.A.F.; SOUZA JÚNIOR, C.L. de. Comparação de índices de seleção não paramétricos para a seleção de cultivares. Bragantia, v.58, p.253-267, 1999. DOI: https://doi.org/10.1590/ S0006-87051999000200005.

KHALID, S.; MALIK, A.U.; SALEEM, B.A.; KHAN, A.S.; KHALID, M.S.; AMIN, M. Tree age and canopy position affect rind quality, fruit quality and rind nutrient content of 'Kinnow' mandarin (Citrus nobilis Lour x Citrus deliciosa Tenora). Scientia Horticulturae, v.135, p.137-144, 2012. DOI: https://doi.org/10.1016/j.scienta.2011.12.010.

LESSA, L.S.; LEDO, C.A. da S.; SANTOS, V. da S. Seleção de genótipos de mandioca com índices não paramétricos. Revista Raízes e Amidos Tropicais, v.13, p.1-17, 2017.

LESSA, L.S.; LEDO, C.A. da S.; SANTOS, V. da S.; SILVA, S. de O. e; PEIXOTO, C.P. Seleção de híbridos diplóides (AA) de bananeira com base em três índices não paramétricos. Bragantia, v.69, p.525-534, 2010. DOI: https://doi.org/10.1590/S000687052010000300003 .

MATTOS JR., D.; DE NEGRI, J.D.; POMPEU JR., J.; GHILARDI, A.A.; AZEVEDO, F.A. de; BASTIANEL, M. Citros: principais informações e recomendações de cultivo. In: AGUIAR, A.T. da E.; GONÇALVES, C.; PATERNIANI, M.E.A.G.Z.; TUCCI, M.L.S.A.; CASTRO, C.E.F. de. (Ed). Instruções agrícolas para as principais culturas econômicas. 7.ed. rev. e atual. Campinas: Instituto Agronômico, 2014. p.140-149. (Boletim IAC, 200).

MULAMBA, N.N.; MOCK, J.J. Improvement of yield potential of the Eto Blanco maize (Zea mays L.) population by breeding for plant traits. Egyptian Journal of Genetics and Cytology, v.7, p.40-51, 1978.

NAVARRO, L.; OLIVARES-FUSTER, O.; JUÁREZ, J.; ALEZA, P.; PINA, J.A.; CERVERA, M.; FAGOAGA, C.; PÉREZ, R.M.; PEÑA, L. Melhoramento de citros na Espanha através da regeneração de triplóides, hibridação somática e transformação genética. In: SEMINÁRIO INTERNACIONAL DE CITROS MELHORAMENTO, 7., 2002, Bebedouro. Anais. Bebedouro: Estação Experimental de Citricultura de Bebedouro, 2002. p.5770. Editores: Luiz Carlos Donadio, Eduardo Sanches Stuchi.

RAGA, V.; BERNET, G.P.; CARBONELL, E.A.; ASINS, M.J. Segregation and linkage analyses in two complex populations derived from the citrus rootstock Cleopatra mandarin. Inheritance of seed reproductive traits. Tree Genetics \& Genomes, v.8, p.1061-1071, 2012. https://doi.org/10.1007/s11295-012-0486-7. 
RAMOS, Y.C.; STUCHI, E.S.; GIRARDI, E.A.; LEÃO, H.C. de; GESTEIRA, A. da S.; PASSOS, O.S.; SOARES FILHO, W. dos S. Dwarfing rootstocks for Valencia sweet orange. Acta Horticulturae. v.1065, p.351-354, 2015. DOI: https://doi.org/10.17660/ActaHortic.2015.1065.42.

SCHINOR, E.H.; CRISTOFANI-YALY, M.; BASTIANEL, M.; MACHADO, M.A. Sunki mandarin vs Poncirus trifoliata hybrids as rootstocks for Pera sweet orange. Journal of Agricultural Science, v.5, p.190-200, 2013. DOI: https://doi.org/10.5539/jas. v5n6p190.

SCHWARZBACH, E. Einige Anwendungsmöglichkeiten elektronischer Datenverarbeitung (EDV) für die Beurteilung von Zuchtmaterial. Pflanzenzüchtertagung Gumpenstein, p.277287, 1972.

SOARES FILHO, W. dos S. (Ed.). Reunião técnica: obtenção, seleção e manejo de variedades porta-enxerto de citros adaptadas a estresses abióticos e bióticos. Cruz das Almas: Embrapa Mandioca e Fruticultura, 2012. (Embrapa Mandioca e Fruticultura. Documentos, 200).
STUCHI, E.S.; DONADIO, L.C.; SEMPIONATO, O.R. Tolerância à seca da laranjeira 'Folha Murcha' em 10 porta-enxertos. Revista Brasileira de Fruticultura, v.22, p.454-457, 2000.

TAZIMA, Z.H.; AULER, P.A.M.; NEVES, C.S.V.J.; YADA, I.F.U.; LEITE JÚNIOR, R.P. Comportamento de clones de laranja 'Valência' na região norte do Paraná. Revista Brasileira de Fruticultura, v.30, p.970-974, 2008. DOI: https://doi.org/10.1590/ S0100-29452008000400022.

VILARINHO, A.A.; VIANA, J.M.S.; SANTOS, J.F. dos; CÂMARA, T.M.M. Eficiência da seleção de progênies s1 e s2 de milho-pipoca, visando à produção de linhagens. Bragantia, v.62, p.9-17, 2003. DOI: https://doi.org/10.1590/S000687052003000100002 .

YACOMELO, M.; BAQUERO, C.; MARTÍNEZ, M.; MURCIA, N.; CORREA, E.; ORDUZ-RODRIGUEZ, J.O. Characterization and selection of Citrus sinensis Osbeck cv. Margaritera parental trees for repopulation in the Mompox depression region, Colombia. Agronomía Colombiana, v.36, p.103-113, 2018. DOI: https://doi.org/10.15446/agron.colomb.v36n2.69634. 